\title{
Conodont biodiversity of the Frasnian-Famennian boundary interval (Upper Devonian) in the Southern Urals
}

\author{
REZEDA C. TAGARIEVA
}

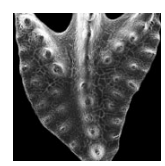

\begin{abstract}
In the sections of the West Uralian Folded Zone (western slope of the South Urals) the boundary between the Frasnian and Famennian (F/F) runs at the bottom of the Barma Horizon. It is established within a lithologically homogeneous brachiopod coquina by simultaneous appearance of the conodont Palmatolepis triangularis Sann. and brachiopod Parapugnax markovskii (Yud.). The brachiopod shell stone of the F/F boundary interval subdivided into the Upper rhenana, linguiformis, Lower-Middle triangularis zones are thoroughly characterized by means of conodonts. In the upper linguiformis Zone at the top of the Frasnian, all the sections show a replacement of the palmatolepid biofacies with its abundant taxa of the genus Palmatolepis by the extremely poor, practically monotaxon icriodid biofacies. This is probably due to changes in the global sea levels and the Mass Extinction Event (Upper Kellwasser Event) at the F/F boundary. $\bullet$ Key words: South Urals, boundary F/F interval, brachiopod coquina, conodonts, brachiopods, biofacies.
\end{abstract}

TAGARIEVA, R.C. 2013. Conodont biodiversity of the Frasnian-Famennian boundary interval (Upper Devonian) in the Southern Urals. Bulletin of Geosciences 88(2), 297-314 (8 figures, 4 tables). Czech Geological Survey, Prague. ISSN 1214-1119. Manuscript received February 3, 2012; accepted in revised form May 2, 2012; published online July 31, 2012; issued June 7, 2013.

Rezeda C. Tagarieva, Russian Academy of Sciences, Ufa Research Centre, Institute of Geology, Karl Marx Street 16/2, Ufa 450000, Russia; stpal@ufaras.ru

The Upper Devonian of the West Uralian Folded Zone (western slope of the South Urals) is subdivided into the Frasnian and Famennian. The Frasnian is represented by the Sargai, Domanik, Mendym and Askyn horizons (Antsygin et al. 1993). The Famennian includes the Barma beds and also the Makarovo, Murzakaevo, Kushelga and Lytva horizons. In many sections (Gabdyuk, Mendym, Zigan and others) there are gaps at the F/F boundary (Abramova 1999, Abramova \& Artyushkova 2004, Veimarn et al. 2004). The duration of the gaps ranges from one zone to several conodont zones. An individual peculiarity of a number of sections (Bol'shaya Barma, Akkyr, Ryauzyak, Kuk-Karauk and Kuktash) is the development of thin (up to $2.1 \mathrm{~m}$ ) brachiopod shell limestones within the boundary F/F interval characterized by a continuous conodont succession (see Fig. 1). In these shell rocks the $\mathrm{F} / \mathrm{F}$ boundary lithologically is unrecognized and determined only by the replacement of conodont and brachiopod assemblages. Faunistically the lower part of the coquinas belongs to the Askyn Horizon of the Frasnian and has a small thickness in all the sections from $0.15 \mathrm{~m}$ to $0.35 \mathrm{~m}$. The thicker upper part of the shell limestones $(0.45-1.78 \mathrm{~m})$ belongs to the Barma beds of the Famennian.

\section{Investigation history of the F/F boundary on the western slope of the South Urals}

The Barma beds were first described by D.V. Nalivkin as the brachiopod limestones with Rhynchonella (Pugnax) triaequalis Goss. at the base of the Famennian Stage (Nalivkin 1926; Fig. 2). They were named after the Bol'shaya Barma River on the western slope of the South Urals (Nalivkin 1931). In 1934-1937 the detailed investigations on Devonian deposits of the Middle and South Urals were carried out by Markovsky (1948). He concluded that the Barma beds should be attributed to the uppermost Askyn Horizon of the Frasnian and represent a brachiopod facies of the upper part of the beds with Hypothyridina cuboides Sow. (see Fig. 2). Domrachev (1952) emphasized the necessity of separating the Barma beds as an individual stratum straton within the top of the Frasnian Stage. He considered the Barma beds to be a typical horizon of regional significance for the Urals. This viewpoint was reflected in subsequent stratigraphic schemes of the Urals (Khodalevich 1968, Breyvel et al. 1980; see Fig. 2).

The very first data on conodonts from the Upper Devonian deposits of the western slope of the South Urals were gained in the late 1980s (Abramova 1999). In all the 


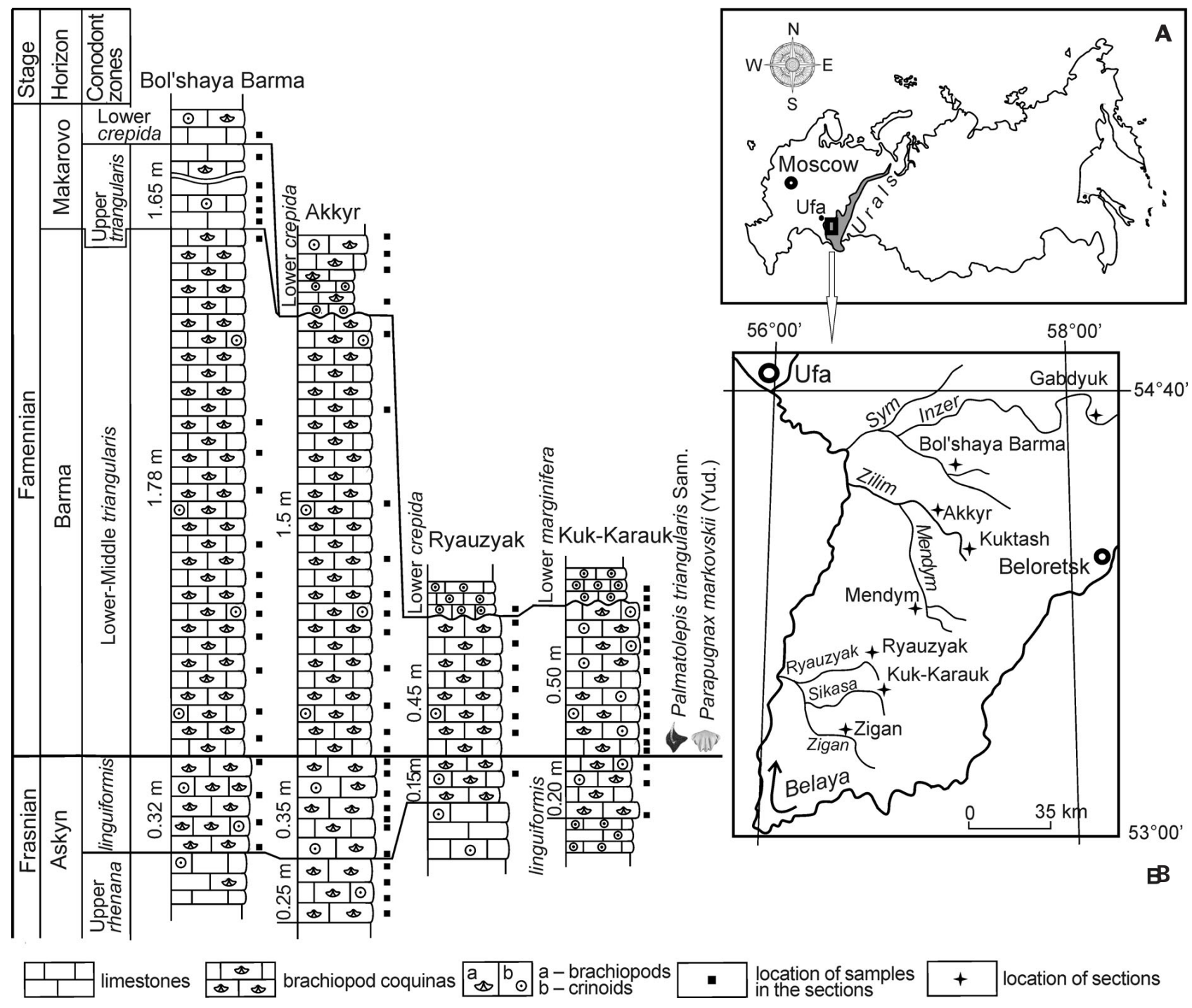

Figure 1. Correlation of sections of the Frasnian-Famennian boundary deposits in the West Uralian Folded Zone (western slope of South Urals). - A - sketch map with the location of the studied area in Russia; B - location of the sections.

sections where the F/F interval consists of brachiopod shell limestones in the form of continuous sequences, A.N. Abramova revealed the simultaneous occurrence emergence of the conodont Palmatolepis triangularis Sann. and brachiopod Parapugnax markovskii (Yud.) (= Pugnoides triaequalis Goss.), the guide fossils for the Barma beds (Abramova 1999, Abramova \& Artyushkova 2004). Therefore, the F/F boundary was established at the bottom of the Barma beds in the stratigraphic scheme accepted by the $4^{\text {th }}$ Ural Stratigraphic Conference (Antsygin et al. 1993; see Fig. 2).

The A.N. Abramova's brachiopod collection from the F/F boundary interval from sections of Bol'shaya Barma and Akkyr was reviewed Mizens $(2007,2009)$. At a time the brachiopod shells from these sections were studied for C-isotope analysis (Izokh \& Mizens 2009). The simulta- neous appearance of Palmatolepis triangularis Sann. and Parapugnax markovskii (Yud.) corresponded with the positive excursions $\delta^{13} \mathrm{C}$ curve were fixed at the base of the Barma Beds in the Bol'shaya Barma section. Whereas the first occurrence of Parapugnax markovskii (Yud.) and higher $\delta^{13} \mathrm{C}$ values in the Akkyr section were marked below the F/F boundary (according to A.N. Abramova's subdivision), i.e., prior to the appearance of Palmatolepis triangularis Sann. (Fig. 3). On the basis of these data A.G. Mizens concluded that the lower part of the Barma beds (about $0.10 \mathrm{~m}$ ) belongs to the Askyn Horizon of the Frasnian.

In order to solve this contradiction in interpreting the stratigraphic framework of the Barma beds and accordingly the location of the F/F boundary, during 2009 and 2010 additional investigations have been conducted on the 
Rezeda C. Tagarieva • Conodont biodiversity of the Frasnian-Famennian boundary interval, Southern Urals

\begin{tabular}{|c|c|c|c|c|c|c|c|c|}
\hline \multirow{2}{*}{$\begin{array}{l}\frac{E}{\Phi} \\
\frac{1}{\omega n} \\
\text { की }\end{array}$} & \multirow{2}{*}{ @ } & \multirow{2}{*}{ 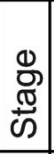 } & \multirow{2}{*}{$\begin{array}{l}\text { Standard } \\
\text { conodont } \\
\text { zonation }\end{array}$} & \multirow[t]{2}{*}{$\begin{array}{l}\text { D.V. Nalivkin } \\
(1926,1931)\end{array}$} & $\begin{array}{l}\text { B.P. Markovsky } \\
\text { (1948) }\end{array}$ & \begin{tabular}{||c||} 
Khodalevich \\
(1968), Breyvel \\
et al. (1980) \\
\end{tabular} & $\begin{array}{c}\text { Antsygin et al. } \\
(1993)\end{array}$ & $\mid$\begin{tabular}{|}
$\mid$ Abramova (1992) \\
Zhamoyda(2008)
\end{tabular} \\
\hline & & & & & Beds & Horizon & Horizon & Horizon \\
\hline \multirow{16}{*}{ 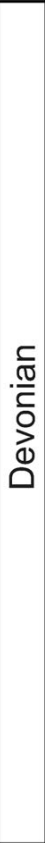 } & \multirow{15}{*}{ 高 } & \multirow{8}{*}{ 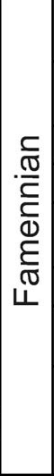 } & praesulcata $\rightarrow$ & \multirow[b]{8}{*}{$\begin{array}{l}\text { The brachiopod } \\
\text { limestones with Pugnax } \\
\text { triaequalis Goss. }\end{array}$} & \multirow{3}{*}{ Ziganian } & \multirow{3}{*}{ Kushelga } & Ziganian beds & \multirow[t]{2}{*}{ Lytva } \\
\hline & & & expansa & & & & Abiyuskanian beds & \\
\hline & & & postera & & & & Kushelga & Kushelga \\
\hline & & & trachytera & & \multirow{2}{*}{\begin{tabular}{|l|} 
Murzakai or beds with \\
Leiorhynchus ursus Nal.
\end{tabular}} & \multirow{2}{*}{ Murzakai } & \multirow[t]{2}{*}{ Murzakai } & \multirow[t]{2}{*}{ Murzakai } \\
\hline & & & \multirow{2}{*}{$\begin{array}{l}\text { marginifera } \\
\text { rhomboidea } \\
\end{array}$} & & & & & \\
\hline & & & & & \multirow{2}{*}{$\begin{array}{l}\text { Makarovo or beds } \\
\text { with Leiorhynchus } \\
\text { polonicus Gür. } \\
\text { (= Zilimia polonica) }\end{array}$} & \multirow[b]{2}{*}{ Makarovo } & \multirow[b]{2}{*}{ Makarovo } & \multirow[b]{2}{*}{ Makarovo } \\
\hline & & & crepida & & & & & \\
\hline & & & triangularis & & $\begin{array}{l}\text { Beds with Pugnoides } \\
\text { triaequalis Goss. }\end{array}$ & Barma & \multirow[t]{2}{*}{ Barma beds } & Barma \\
\hline & & \multirow{7}{*}{$\begin{array}{l}\frac{c}{\frac{0}{c}} \\
\frac{1}{0} \\
\frac{\pi}{4} \\
\frac{1}{4}\end{array}$} & linguiformis & \multirow[t]{8}{*}{$\begin{array}{l}\text { Beds with Hypothyridina } \\
\text { cuboides Sow. }\end{array}$} & \multirow{2}{*}{\begin{tabular}{|l|}
$\begin{array}{l}\text { Beds with Hypothyridina } \\
\text { cuboides Sow. }\end{array}$ \\
Mendym
\end{tabular}} & Askyn & & Askyn \\
\hline & & & rhenana & & & Mendym & Mendym & Mendym \\
\hline & & & jamieae & & \multirow{3}{*}{ Domanik } & \multirow{3}{*}{ Domanik } & \multirow{3}{*}{ Domanik } & \multirow{3}{*}{ Domanik } \\
\hline & & & hassi & & & & & \\
\hline & & & \multirow{2}{*}{\begin{tabular}{|l|} 
punctata \\
transitans \\
\end{tabular}} & & & & & \\
\hline & & & & & \multirow{3}{*}{$\begin{array}{l}\text { "Sub-Domanik" or Sargai } \\
\text { beds with Hypothyridina } \\
\text { calva Mark. }\end{array}$} & Sorrai & Sorroi & Sorani \\
\hline & & & falsiovalis & & & Gargar & vargar & Jargar \\
\hline & Mid. & Giv. & & & & Kyn & Kyn & Kyn \\
\hline
\end{tabular}

Figure 2. Investigation history of the F/F boundary on the western slope of the South Urals.

controversial interval with centimetre by centimetre sampling for conodonts. The A.N. Abramova original field materials, and the conodont collection studied by V.N. Basyshev in the 1980's were re-examined. It was found out that the interval $0.25 \mathrm{~m}$ thick in which sample 7123 was collected (see Fig. 3) corresponds in fact to the uppermost part of the linguiformis Zone $(0.17 \mathrm{~m})$ whereas the upper part of the interval $0.08 \mathrm{~m}$ thick is already of Famennian age (Tagarieva 2011). It is precisely in this interval that the first Parapugnax markovskii (Yud.) make their appearance.

Sharing the opinions of previous authors, it sounds reasonable to rank the Barma beds as a biostratigraphic horizon. This is actually a faunistically recognizable independent stratum characterizing a definite stratigraphic interval within the scope of the conodont Lower-Middle triangularis zones (Abramova 1992, Baryshev \& Abramova 1996, Abramova \& Artyushkova 2004, Artyushkova et al. 2011b).

\section{Material and methods}

Stone material from the Frasnian/Famennian boundary interval was collected during field works of 2009-2011 from four sections (Bol'shaya Barma, Akkyr, Ryauzyak and Kuk-Karauk). Investigations of the sections were perfor- med in the most detailed way with centimetre-bycentimetre simultaneous sampling for conodonts and brachiopods. A total of more than 60 samples were collected. The weight of each sample did not exceed $1.5 \mathrm{~kg}$. Brachiopods were separated first before the laboratory treatment of the samples. The rest of the rock $(0.3 \mathrm{~kg}$ on the average) was used for separating conodonts. To do this a 3-5 percent solution of formic acid was used. All the samples under investigation, despite their small weight, yielded conodont remains. The collection includes about 4133 platform elements belonging to 57 species of seven genera. The collections stored in the Laboratory of Paleozoic Stratigraphy, Institute of Geology, Ufa Research Centre, Russian Academy of Sciences. Conodont photographs were made by means of scanning electron microscopy in the A.A. Trofimuk Institute of Petroleum Geology and Geophysics, Siberian Branch of the Russian Academy of Sciences (Novosibirsk) and in the A.A. Borisyak Paleontological Institute of the Russian Academy of Sciences (Moscow).

\section{Brief description of lithology and conodont distribution of the F/F boundary interval in the sections}

In the sections that have been studied on the western slope of the South Urals Upper Frasnian and Lower Famennian 


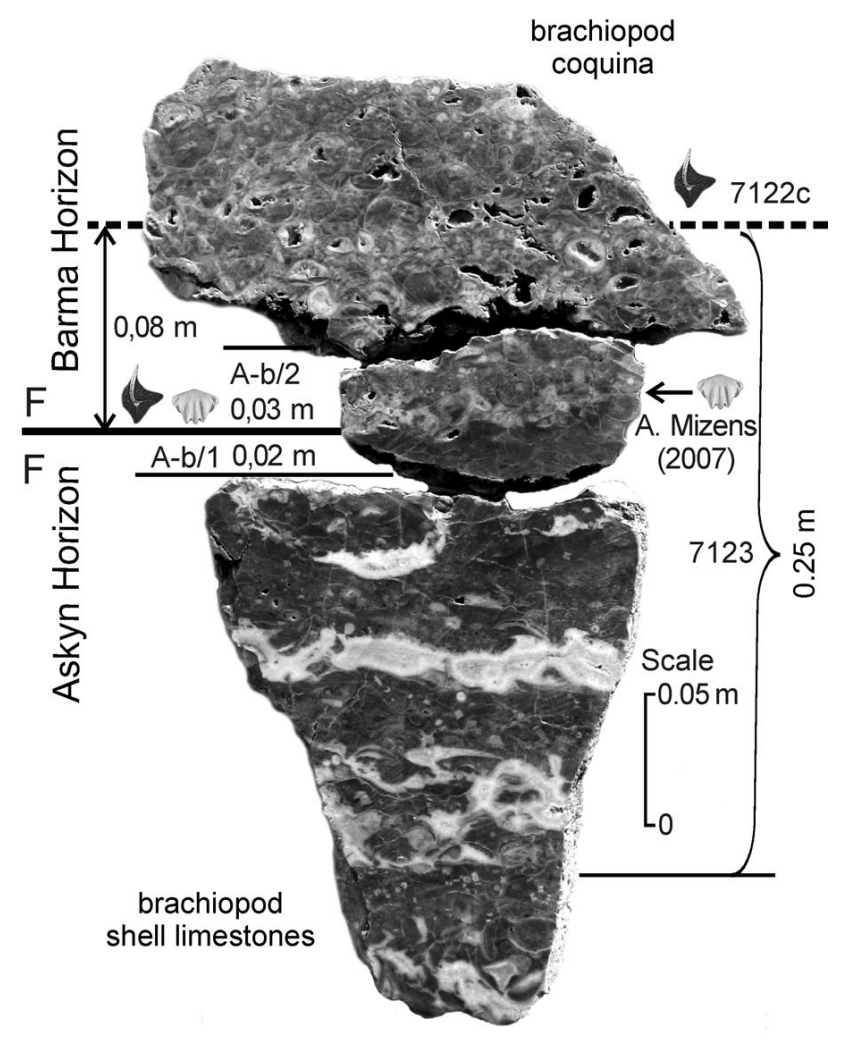

..-- position of the F/F boundary according

to A.N. Abramova \& O.V. Artyushkova (2004)

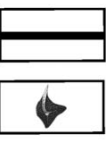

position of the F/F boundary,

present paper (Tagarieva 2011)

Palmatolepis triangularis Sann.

(UP) Parapugnax markovskii (Yud.)

A-b/1 samples (Tagarieva 2011)

7123 samples (Abramova \& Artyushkova 2004)

Figure 3. Polished slab of a brachiopod shell limestones from the F/F boundary interval, the Akkyr section.

deposits are characterized by relatively deep shelf facies (Abramova \& Artyushkova 2004). A detailed investigation on the Frasnian deposits of the upper Askyn Horizon, and on the Famennian Barma and Makarovo horizons has been carried out.

The Askyn Horizon is usually represented by light-grey and white massive and thick-layered organogenic limestones with abundant brachiopods that form coquinas at some places. The overlying shell limestones of the Barma Horizon of the Famennian occur with a gradational transition. They consist of light-grey limestones overfilled with brachiopods. Above the Barma Horizon the fossil record succession interrupts in most of the sections. For this reason the lower part of the Makarovo deposits is absent from many sections. The Makarovo rocks are light-grey and

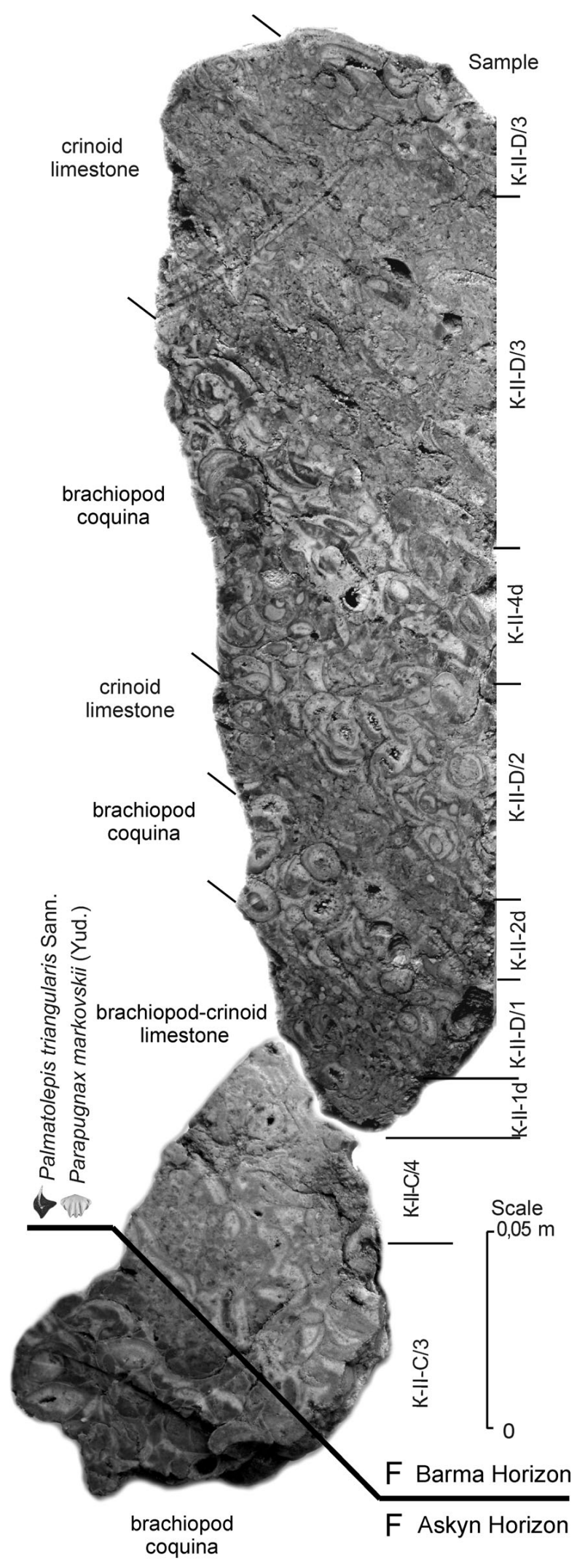

Figure 4. Polished slab of a brachiopod shell stones from the F/F boundary interval, the Kuk-Karauk section. Alternation of beds rich in crinoid detritus and brachiopod varieties in Barma limestones. 
Table 1. Numerical distribution of conodont taxa in samples from the Bol'shaya Barma section. For the position of samples in the section see Fig. 1. The samples of the Barma brachiopod coquina yield a conodont assemblage that contanins, together with Famennian conodonts, Frasnian species of the genus Palmatolepis, which are found in Askyn Horizon (inside the oval). The following abbreviations for conodont genera and species are used throughout the tables and the text paper: Ad. - Ancyrodella; Anc. - Ancyrognathus; B. - Belodella; Ic. -Icriodus; Pa. - Palmatolepis; Pol. - Polygnathus; a-alternatus; $d$-delicatula; $i$-iowaensis; $q$ - quadratinodosalobata; $p$ - perlobata.

\begin{tabular}{|c|c|c|c|c|c|c|c|c|c|c|c|c|c|c|c|c|c|c|c|c|c|c|c|}
\hline Stage & \multirow{2}{*}{\multicolumn{4}{|c|}{$\begin{array}{c}\text { Frasnian } \\
\text { Askyn }\end{array}$}} & \multicolumn{19}{|c|}{ Famennian } \\
\hline Horizon & & & & & \multicolumn{12}{|c|}{ Barma } & \multicolumn{7}{|c|}{ Makarovo } \\
\hline Conodont zone & \multicolumn{4}{|c|}{ linguiformis } & \multicolumn{12}{|c|}{ Lower-Middle triangularis } & \multicolumn{6}{|c|}{ Upper triangularis } & \begin{tabular}{|l|} 
Lower \\
crepidal
\end{tabular} \\
\hline Meters (m) & \multicolumn{4}{|c|}{0.32} & \multicolumn{12}{|c|}{1.78} & \multicolumn{6}{|c|}{1.65} & \\
\hline Weight (kg) & 0.4 & 1.3 & 1.0 & 0.3 & 0.2 & 0.8 & 0.5 & 0.3 & 0.3 & 0.7 & 0.4 & 0.6 & 0.8 & 0.3 & 0.3 & 0.5 & 0.2 & 0.3 & 0.3 & 0.5 & 0.2 & 0.3 & 0.4 \\
\hline Sample № & $\frac{n}{\dot{m}}$ & 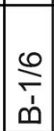 & $\stackrel{N}{\stackrel{1}{n}}$ & $\frac{\infty}{\stackrel{\infty}{m}}$ & $\begin{array}{l}\bar{T} \\
\hat{1} \\
\sim \\
\sim \\
0\end{array}$ & \begin{tabular}{|l|}
$N$ \\
$N$ \\
10 \\
$N$ \\
0 \\
0
\end{tabular} & 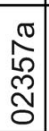 & 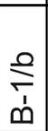 & $\frac{0}{\stackrel{c}{m}}$ & $\frac{\text { co }}{\frac{1}{m}}$ & $\begin{array}{l}\mathbb{D} \\
\stackrel{N}{ } \\
\tilde{\omega}\end{array}$ & $\begin{array}{l}m \\
m\end{array}$ & $\begin{array}{l}\text { ț } \\
\text { m. }\end{array}$ & $\begin{array}{l}p_{1} \\
\dot{m}\end{array}$ & $\begin{array}{l}\varphi \\
\dot{\omega}\end{array}$ & 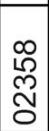 & $\underset{\infty}{\stackrel{N}{N}}$ & $\left|\begin{array}{l}0 \\
\mathbb{N} \\
\infty\end{array}\right|$ & 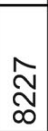 & $\underset{\infty}{\stackrel{N}{N}}$ & $\begin{array}{l}\mathscr{m} \\
\tilde{m} \\
\infty\end{array}$ & $\mid \begin{array}{c}N \\
\sim \\
\sim \\
\infty\end{array}$ & 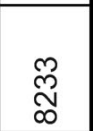 \\
\hline Pa. amplificata Klapp., Kuzm. \& Ovn. & 2 & 1 & 1 & & & & & & & & & & & & & & & & & & & & \\
\hline Pa. kireevae Ovn. & 2 & & & & & & & & & & & & & & & & & & & & & & \\
\hline Pa. linguiformis Müll. & 1 & 1 & 1 & 2 & & & (1) & & & & & & & & & & & & & & & & \\
\hline Pa. ljaschenkoae Ovn. & 1 & 1 & 3 & 1 & & & & & & & & & & & & & & & & & & & \\
\hline Pa. nasuta Müll. & 1 & & 4 & 6 & & & & & & & & & & & & & & & & & & & \\
\hline Pa. rhenana Bisch. & 1 & & & 1 & (aff) & & (aff) & & & (aff) & & & & & & & & & & & & & \\
\hline Pa. subrecta Mill. \& Young. & 4 & 5 & 7 & 4 & (1) & & & & & & & & & & & & & & & & & & \\
\hline Pol. krestovnikovi Ovn. & 3 & 2 & 10 & 4 & & & & & & & & & & & & & & & & & & & \\
\hline Pol. siratchoicus Ovn. \& Kuzm. & 1 & 1 & & & & & & & & & & & & & & & & & & & & & \\
\hline Pol. uchtensis Ovn. \& Kuzm. & 1 & 2 & & & & & & & & & & & & & & & & & & & & & \\
\hline Pa. ederi Zieg. \& Sand. & & & 1 & 1 & & & & & & & & & & & & & & & & & & & \\
\hline Pa. gigas extensa Zieg. \& Sand. & & & 2 & 2 & & & & (1) & (1) & & & (aff) & & & & & & & & & & & \\
\hline Pa. hassi Müll. \& Müll. & & & 1 & 2 & (1) & & & & & & & & & & & & & & & & & & \\
\hline Pol. brevilaminus $\mathrm{Br}$ \& M. & 2 & 9 & 9 & 6 & 2 & 10 & 2 & 1 & 3 & 6 & 4 & 2 & 1 & 3 & 4 & 1 & 2 & 4 & 1 & 1 & 1 & 20 & 5 \\
\hline Pa. praetriangularis Zieg. \& Sand. & & & & & 3 & 2 & 4 & 1 & 1 & & & & & & & & & & & & & & \\
\hline Pa. triangularis Sann. & & & & & 4 & 17 & 1 & 2 & 2 & 6 & 10 & 5 & 11 & 7 & 6 & 5 & 4 & 5 & 3 & 2 & 6 & 42 & 14 \\
\hline Pa. d. delicatula Br. \& M. & & & & & 1 & & 1 & & 1 & 2 & & & & 3 & & & 3 & & & & 2 & 1 & 1 \\
\hline Ic. alternatus alternatus $\mathrm{Br}$. \& M. & & & & & 2 & 11 & & & & 1 & 2 & & & & & 4 & & 3 & 8 & 1 & 1 & 10 & 11 \\
\hline Ic. alternatus helmsi Sand. & & & & & 1 & 7 & & & & 1 & 2 & 3 & & & & & & & & & & 4 & \\
\hline Pa. clarki Zieg. & & & & & & & & 1 & & 6 & 6 & & & 4 & 2 & 3 & 2 & 4 & 1 & & 1 & 8 & 14 \\
\hline Pa. q. praeterita Schül. & & & & & & & & & 1 & 4 & 2 & 7 & 4 & 2 & & 5 & 1 & 3 & & & & 3 & 1 \\
\hline Ic. i. iowaensis Youn. \& Pet. & & & & & & & & & & & & 1 & & $\begin{array}{ll}2 \\
1\end{array}$ & 2 & 8 & 4 & & & & & 4 & \\
\hline Pol. izhmensis Kuzm. & & & & & & & & & & & & 1 & 1 & 1 & 2 & & & & & & & & \\
\hline Pol. subinornatus Strel. & & & & & & & & & & & & & 3 & 2 & 1 & 4 & & & & & & 2 & 10 \\
\hline Pa. subperlobata Br. \& M. & & & & & & & & & & & & & 1 & & 2 & & & & & & 2 & 2 & 3 \\
\hline Pol. praecursor Mat. & & & & & & & & & & & & & 1 & 1 & & & 3 & 2 & 2 & & & & \\
\hline Pa. spathula Schül. & & & & & & & & & & & & & & & & & 1 & 2 & & & & & 2 \\
\hline Pa. tenuipunctata Sann. & & & & & & & & & & & & & & & & & 1 & & & & 2 & & 10 \\
\hline Pa. p. perlobata UI. \& Bas. & & & & & & & & & & & & & & & & & 1 & & 1 & & 1 & & 1 \\
\hline Pa. minuta loba Helms & & & & & & & & & & & & & & & & & & & & & & & 1 \\
\hline Pa. q. sandbergi Ji \& Zieg. & & & & & & & & & & & & & & & & & & & & & & & 3 \\
\hline
\end{tabular}

pinkish-grey, thin and medium-layered organogenic limestones with brachiopods, crinoids, rugose corals and other faunas (Abramova 1999, Artyushkova et al. 2011a). Brachiopod shell stones of the F/F boundary interval are subdivided into the four conodont Upper rhenana, linguiformis (Frasnian, Askyn Horizon) and Lower-Middle triangularis (Famennian, Barma Horizon) zones (Abramova 1999, Abramova \& Artyushkova 2004). The division is performed in terms of the standard conodont zonation (Ziegler \& Sandberg 1990). The description of typical (Bol'shaya Barma and Akkyr) and peculiar (Ryauzyak and Kuk-Karauk) sections, including a short description of the conodont faunas is given below.
The Bol'shaya Barma section. - The section on the Bol'shaya Barma River (right tributary of the Askyn River) serves as a stratotype of the Barma Horizon. It is located on the right bank of the river of the same name $1.3 \mathrm{~km}$ upstream from its mouth (see Fig. 1A, B). The brachiopod shell limestone thickness in the $\mathrm{F} / \mathrm{F}$ interval is $2.1 \mathrm{~m}$. The lower part of these shell stones $0.32 \mathrm{~m}$ thick belongs to the Askyn Horizon of the Frasnian while the upper part $1.78 \mathrm{~m}$ thick corresponds to the Barma Horizon of the Famennian.

The Frasnian portion of the shell stones consists of light-grey compact limestones with interbeds rich in brachiopods where shells are unsorted by size and distributed 
Table 2. Numerical distribution of conodont taxa in samples from the Akkyr section. For the position of samples in the section see Fig. 1.

\begin{tabular}{|c|c|c|c|c|c|c|c|c|c|c|c|c|c|c|c|c|c|c|c|c|c|c|c|c|c|c|}
\hline Stage & \multirow{2}{*}{\multicolumn{11}{|c|}{$\begin{array}{l}\text { Frasnian } \\
\text { Askyn }\end{array}$}} & \multicolumn{15}{|c|}{ Famennian } \\
\hline Horizon & & & & & & & & & & & & & & & & & & arn & & & & & & & lakar & rovo \\
\hline Conodont zone & & $\begin{array}{l}\text { Up } \\
\text { rhen }\end{array}$ & $\begin{array}{l}\text { per } \\
\text { nan }\end{array}$ & & & & ing & uifo & $r m$ & & & & & & we & $r-M$ & Mido & dle & tric & ange & ula & & & & $\begin{array}{l}\text { owe } \\
\text { repic }\end{array}$ & \\
\hline Meters (m) & & & 25 & & & & & 0.35 & & & & & & & & & & 50 & & & & & & & & \\
\hline Weight $(\mathrm{kg})$ & 0.3 & & 0.2 & 0.2 & 0.7 & & 0.6 & 0.2 & 0.7 & 1.9 & 0.3 & 0.3 & 1.6 & 1.5 & 1.0 & 1.0 & 0.6 & 0.8 & 0.7 & 0.5 & 1.5 & 0.5 & 0.5 & 1.2 & \begin{tabular}{|l|l|}
2 & 0.7 \\
\end{tabular} & 0.6 \\
\hline Taxon Sample № & 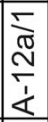 & 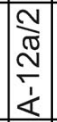 & 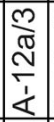 & 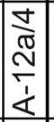 & 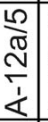 & 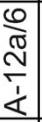 & 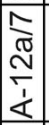 & 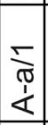 & $\frac{N}{\frac{N}{\alpha}}$ & $\frac{m}{\infty}$ & $\frac{5}{\frac{1}{4}}$ & $\frac{N}{\frac{N}{1}}$ & 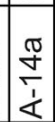 & $\begin{array}{c}\mathbb{R} \\
\stackrel{0}{0} \\
\frac{1}{<} \\
\end{array}$ & $\begin{array}{l}\mathbb{0} \\
\stackrel{0}{1} \\
1 \\
\end{array}$ & $\mid \begin{array}{c}\pi \\
\frac{\pi}{1} \\
\frac{1}{4} \\
\end{array}$ & $\frac{0}{\frac{1}{2}}$ & $\begin{array}{c}\infty \\
\infty \\
\frac{1}{\alpha} \\
\end{array}$ & $\frac{0}{\frac{\infty}{5}}$ & $\mid \begin{array}{c}0 \\
\infty \\
\frac{1}{1} \\
\end{array}$ & $\begin{array}{c}2 \\
\infty \\
\frac{1}{1} \\
\end{array}$ & $\frac{\infty}{\frac{\infty}{\alpha}}$ & & $\frac{\pi}{2}$ & $\frac{2}{\frac{0}{1}}$ & 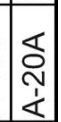 \\
\hline Ad. ioides Zieg. & 3 & & & 4 & & & & & & & & & & & & & & & & & & & & & & \\
\hline Ad. nodosa UI. \& Bas. & 1 & 4 & 6 & 11 & 1 & & & & & & & & & & & & & & & & & & & & & \\
\hline Belodella sp. & 5 & 2 & & 1 & & & & & & & & & & & & & & & & & & & & & & \\
\hline Pa. kireevae Ovn. & 1 & & 1 & & & & & & & & & & & & & & & & & & & & & & & \\
\hline Pa. ljaschenkoae Ovn. & 1 & 1 & 2 & 5 & & & & & & & & & & & & & & & & & & & & & & \\
\hline Pa. nasuta Müll. & 23 & 24 & 23 & 107 & 106 & & 8 & & 2 & 15 & 11 & & & & & & & & & & & & & & & \\
\hline Pa. rhenana Bisch. & 3 & 1 & 2 & 12 & 34 & 1 & 1 & 1 & & & & & & & & & & & & & & & & & & \\
\hline Pa. subrecta Mill. \& Young. & 5 & 10 & & 20 & 67 & & & & 1 & & & & & & & & & & & & & & & & & \\
\hline Pol. siratchoicus Ovn. \& Kuzm. & 2 & 3 & 3 & 10 & 20 & & & & & & & & & & & & & & & & & & & & & \\
\hline Pa. orlovi Khrus. \& Kuzm. & & 1 & & & & & 2 & & 1 & & & & & & & & & & & & & & & & & \\
\hline Pa. hassi Müll. \& Müll. & & & 1 & $3 \mid$ & 3 & & 2 & 3 & & 2 & 5 & & & & & & & & & & & & & & & \\
\hline Pa. jamieae Zieg. \& Sand. & & & & 7 & 88 & & & & & & & & & & & & & & & & & & & & & \\
\hline Pa. juntianensis Han & & & & 11 & 11 & & & & & & & & & & & & & & & & & & & & & \\
\hline Pa. mucronata Klapp., Kuzn & & & & 1 & 1 & 1 & & & & & & & & & & & & & & & & & & & & \\
\hline Pa. amplificata Klapp., Kuzn & & & & & 20 & & & & & & 2 & & & & & & & & & & & & & & & \\
\hline Pa. brevis Zieg. \& Sand. & & & & & 9 & & & & & & & & & & & & & & & & & & & & & \\
\hline Pa. gigas extensa Zieg. \& Sand. & & & & & 3 & & 2 & & 1 & 1 & 1 & & & & & & & & & & & & & & & \\
\hline Pa. gigas gigas Mill. \& Young. & & & & & 5 & & & & & 1 & & & & & & & & & & & & & & & & \\
\hline Pa. linguiformis Müll. & & & & & 5 & & & & & & & & & & & & & & & & & & & & & \\
\hline Pa. aff. nicolli Klapp. & & & & & 3 & & & & & & & & & & & & & & & & & & & & & \\
\hline Palmatolepis sp. & & & & & 2 & & & & & & & & & & & & & & & & & & & & & \\
\hline Pol. macilentus Ovn. \& Kuzm. & & & & & 3 & & & & & & & & & & & & & & & & & & & & & \\
\hline Pol. uchtensis Ovn. \& Kuzm. & & & & & 3 & & & & & & & & & & & & & & & & & & & & & \\
\hline Pol. krestovnikovi Ovn. & & & & & & 4 & 7 & 5 & 6 & 5 & 1 & & & & & & & & & & & & & & & \\
\hline Ad. curvata (Br. \& M.) & & & & & & & 2 & & & & & & & & & & & & & & & & & & & \\
\hline Pol. webbi Stauf. & & & & & & & 1 & & & & & & & & & & & & & & & & & & & \\
\hline Ic. alternatus alternatus $\mathrm{Br}$ \& \& M. & & & & & 3 & 10 & 18 & 3 & 8 & 9 & 18 & 4 & 74 & 22 & 7 & 5 & 7 & 3 & 2 & 5 & 54 & 45 & 12 & 3 & 310 & 7 \\
\hline Ic. alternatus helmsi Sand. & & & & & & & 2 & 2 & & & 5 & 1 & 6 & & & & & & & & 8 & 10 & & & & \\
\hline Ic. iowaensis iowaensis Youn. \& Pet. & & & & & & & & & 1 & 3 & 11 & 7 & 10 & 2 & & & & 1 & & & 3 & 1 & 1 & & & \\
\hline Pol. planarius Klapp. \& Lane & & & & & & & & & 1 & 2 & 1 & & & & & & & & & & & & & & & \\
\hline Ancyrognathus sp. & & & & & & & & & 1 & 3 & 1 & & & & & & & & & & & & & & & \\
\hline Pol. brevilaminus $\mathrm{Br}$. \& M. & & & & & & & & & & 3 & 6 & 8 & 5 & 1 & & & 4 & 1 & 2 & & 7 & 4 & & 2 & & 2 \\
\hline Pa. praetriangularis Zieg. \& Sand. & & & & & & & & & & & & 2 & 2 & & & & & & & & & & & & & \\
\hline Pa. triangularis Sann. & & & & & & & & & & & & 2 & 7 & 2 & 1 & & 1 & 1 & 1 & 3 & 15 & 3 & & & & 2 \\
\hline Pa. clarki Zieg. & & & & & & & & & & & & & & & & 2 & 2 & $2 \mid$ & 1 & 1 & & & & & & \\
\hline Pa. d. delicatula Br. \& M. & & & & & & & & & & & & & & & & & 1 & 1 & & & & & & & 2 & 2 \\
\hline Pa. q. praeterita Schül. & & & & & & & & & & & & & & & & 2 & 2 & 1 & & 2 & 10 & 5 & 2 & & & 2 \\
\hline Pelekysgnathus sp. & & & & & & & & & & & & & & & & & & 2 & & & & & & & & \\
\hline Pol. praecursor Mat. & & & & & & & & & & & & & & & & 1 & 2 & 2 & 1 & & 2 & 1 & & 2 & & 20 \\
\hline Pol. subinornatus Strel. & & & & & & & & & & & & & & & & 1 & & 2 & & & & & & & 2 & 2 \\
\hline Pa. subperlobata Br. \& M. & & & & & & & & & & & & & & & & & & & 1 & & & 2 & 1 & & 3 & 4 \\
\hline Pa. p. perlobata UI. \& Bas. & & & & & & & & & & & & & & & & & & & & & & & & & 2 & 4 \\
\hline Pa. aff. circularis Szulc. & & & & & & & & & & & & & & & & & & & & & & & & & 2 & \\
\hline Pa. q. sandbergi Ji \& Zieg. & & & & & & & & & & & & & & & & & & & & & & & & & 1 & \\
\hline Pa. spathula Schül. & & & & & & & & & & & & & & & & & & & & & & & & & 1 & \\
\hline
\end{tabular}

unevenly in the surrounding rock. The space among brachiopods is filled with fine detritus composed mainly of crinoid segments. The established conodont assemblage is characteristic of the linguiformis Zone the nominal species Palmatolepis linguiformis Müll. appears at the base of the brachiopod shell stones. The number of specimens for samples is only 1 or 2 . The conodont assemblage includes not many taxa and consists of 2 genera and 14 species (Table 1). The conodonts Palmatolepis nasuta Müll., Pa. subrecta Mill. \& Young., Polygnathus brevilaminus Br. \& M. and Polygnathus krestovnikovi Ovn. quantitatively surpass the other species. 
Table 3. Numerical distribution of conodont taxa in samples from the Ryauzyak section. For the position of samples in the section see Fig. 1.

\begin{tabular}{|c|c|c|c|c|c|c|c|c|c|}
\hline Stage & \multirow{2}{*}{\multicolumn{2}{|c|}{$\begin{array}{c}\text { Frasnian } \\
\text { Askyn }\end{array}$}} & \multicolumn{7}{|c|}{ Famennian } \\
\hline Horizon & & & \multicolumn{6}{|c|}{ Barma } & Makarovo \\
\hline Conodont zone & \multicolumn{2}{|c|}{ linguiformis } & \multicolumn{6}{|c|}{ Lower-Middle triangularis } & $\begin{array}{l}\text { Lower } \\
\text { crepida }\end{array}$ \\
\hline Meters (m) & \multicolumn{2}{|c|}{0.15} & \multicolumn{6}{|c|}{0.45} & \\
\hline Weight (kg) & 1.0 & 0.4 & 0.3 & 0.2 & 0.3 & 0.2 & 0.2 & 0.2 & 0.6 \\
\hline Taxon Sample № & $P-b$ & P-2 & $\mathrm{P}-\mathrm{C}$ & $P-3$ & $\mathrm{P}-4$ & $\mathrm{P}-\mathrm{e}$ & $\mathrm{P}-5$ & $\mathrm{P}-5 \mathrm{a}$ & $P-6$ \\
\hline Ad. curvata (Br. \& M.) & 3 & & & & & & & & \\
\hline Ad. gigas Young. & 5 & & & & & & & & \\
\hline Ad. nodosa UI. \& Bas. & 3 & & & & & & & & \\
\hline Belodella sp. & 29 & & & & & & & & \\
\hline Pa. amplificata Klapp., Kuzm. \& Ovn. & 18 & & & & & & & & \\
\hline Pa. ederi Zieg. \& Sand. & 1 & & & & & & & & \\
\hline Pa. gigas extensa Zieg. \& Sand. & 39 & & & & & & & & \\
\hline Pa. hassi Müll. \& Müll. & 12 & & & & & & & & \\
\hline Pa. juntianensis Han & 6 & & & & & & & & \\
\hline Pa. kireevae Ovn. & 3 & 1 & & & & & & & \\
\hline Pa. linguiformis Müll. & 7 & & & & & & & & \\
\hline Pa. ljaschenkoae Ovn. & 2 & & & & & & & & \\
\hline Pa. nasuta Müll. & 70 & 4 & & & & & & & \\
\hline Pa. rhenana Bisch. & 15 & & & & & & & & \\
\hline Pa. subrecta Mill. \& Young. & 2 & & & & & & & & \\
\hline Pol. krestovnikovi Ovn. & 26 & 2 & & & & & & & \\
\hline Pol. macilentus Ovn. \& Kuzm. & 18 & & & & & & & & \\
\hline Pol. siratchoicus Ovn. \& Kuzm. & 15 & 1 & & & & & & & \\
\hline Pol. uchtensis Ovn. \& Kuzm. & 6 & 1 & & & & & & & \\
\hline Ic. alternatus alternatus $\mathrm{Br}$ \& $\mathrm{M}$. & 15 & 25 & 36 & 63 & 4 & 7 & 17 & 16 & 2 \\
\hline Pol. brevilaminus Br. \& M. & 5 & & 3 & 12 & & 2 & & 5 & 1 \\
\hline Ic. alternatus helmsi Sand. & & 2 & 2 & 3 & & & & 1 & \\
\hline Pa. praetriangularis Zieg. \& Sand. & & & 1 & 2 & & & & & \\
\hline Pa. triangularis Sann. & & & 3 & 15 & 1 & 1 & 3 & 3 & 2 \\
\hline Ic. i. iowaensis Youn. \& Pet. & & & & & & 1 & 3 & & \\
\hline Pol. izhmensis Kuzm. & & & & & & 1 & & & \\
\hline Pa. clarki Zieg. & & & & & & & 2 & 3 & 8 \\
\hline Pa. q. praeterita Schül. & & & & & & & 1 & & 13 \\
\hline Pa. subperlobata Br. \& M. & & & & & & & 1 & 1 & 8 \\
\hline Pol. subinornatus Strel. & & & & & & & 3 & 2 & 9 \\
\hline Pa. circularis Szulc. & & & & & & & & & 11 \\
\hline Pa. q. sandbergi Ji \& Zieg. & & & & & & & & & 21 \\
\hline Pa. spathula Schül. & & & & & & & & & 2 \\
\hline
\end{tabular}

The Barma brachiopod shell limestone encompasses the interval of the joint conodont Lower-Middle triangularis zones. It differs from the underlying Frasnian shell limestone by a more even and close "packing" of brachiopods. The very first rare specimens of the conodont Palmatolepis triangularis Sann. and brachiopod Parapugnax markovskii (Yud.) have been found in a single sample and their appearance determines the base of the Barma Horizon. The nominal species is together with Icriodus alternatus alternatus Br. \& M., Ic. alternatus helmsi Sand., Ic. iowaensis iowaensis Young. \& Pet., Pa. delicatula delicatula Br. \& M., Pa. praetriangularis Zieg. \& Sand. (see Table 1). Polygnathus brevilaminus Br. \& M. pass through the Famennian. Scarce specimens of the species Palmatolepis clarki Zieg., Pa. quadratinodosalobata praeterita Schül., Pa. subperlobata Br. \& M., Polygnathus izhmensis Kuzm., Pol. praecursor Mat., Pol. subinornatus Strel. appear in the $0.42 \mathrm{~m}$ above base of the Barma shell limestone (see Table 1). It should be noted that the Barma conodont assemblage contains, together with Famennian species, solitary (1 or 2 specimens) Palmatolepis gigas extensa Zieg. \& Sand., Pa. rhenana Bisch., Pa. hassi Müll. \& Müll., Pa. subrecta Mill. \& Young., Pa. linguiformis Müll., Pa. aff. rhenana Bisch. characteristic of the uppermost Askyn Horizon of the Frasnian (see Table 1).

As compared to the other sections, the Famennian portion of the brachiopod coquina (Barma Horizon) has the following peculiarities in the Bol'shaya Barma section. The Barma Horizon's a maximum thickness is $1.78 \mathrm{~m}$ in this section. The taxa of the genus Icriodus are found only at the base of the Famennian, and their number is 
Table 4. Numerical distribution of conodont taxa in samples from the Kuk-Karauk section. For the position of samples in the section see Fig. 1.

\begin{tabular}{|c|c|c|c|c|c|c|c|c|c|c|c|c|c|c|c|c|c|}
\hline Stage & \multicolumn{3}{|c|}{ Frasnian } & \multicolumn{12}{|c|}{ Famennian } & & \\
\hline Horizon & \multicolumn{3}{|c|}{ Askyn } & \multicolumn{12}{|c|}{ Barma } & \multicolumn{2}{|c|}{ Makarovo } \\
\hline Conodont zone & \multicolumn{3}{|c|}{ linguiformis } & \multicolumn{12}{|c|}{ Lower-Middle triangularis } & \multicolumn{2}{|c|}{$\begin{array}{l}\text { Lower } \\
\text { marginifera }\end{array}$} \\
\hline Meters (m) & \multicolumn{3}{|c|}{0.20} & \multicolumn{12}{|c|}{0.50} & & \\
\hline Weight $(\mathrm{kg})$ & 0.4 & 0.2 & 0.3 & 0.4 & 0.2 & 0.2 & 0.1 & 1.0 & 0.3 & & & 0.1 & 0.1 & 0.3 & 0.1 & 0.3 & 0.4 \\
\hline Taxon $\quad$ Sample № & $\frac{0}{\stackrel{1}{\overline{1}}}$ & $\begin{array}{l}\frac{5}{d} \\
\frac{1}{\bar{x}} \\
\underline{\underline{x}}\end{array}$ & 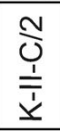 & $\begin{array}{l}\frac{m}{0} \\
\dot{1} \\
\overline{\bar{x}}\end{array}$ & 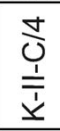 & 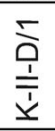 & $\frac{\stackrel{i}{N}}{\overline{\frac{1}{亠}}}$ & 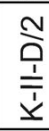 & $\frac{1}{\dot{q}}$ & & & 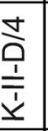 & 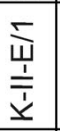 & 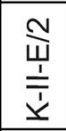 & $\frac{m}{\stackrel{m}{\frac{1}{亠}}}$ & 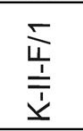 & $\begin{array}{l}\frac{N}{\mathbf{N}} \\
\frac{1}{\bar{亠}} \\
\underline{\underline{T}}\end{array}$ \\
\hline Pa. amplificata Klapp., Kuzm. \& Ovn. & 2 & & 1 & & & & & & & & & & & & & & \\
\hline Pa. nasuta Müll. & 4 & 8 & 1 & & & & & & & & & & & & & & \\
\hline Pol. krestovnikovi Ovn. & 1 & 2 & 2 & & & & & & & & & & & & & & \\
\hline Pol. uchtensis Ovn. \& Kuzm. & 1 & & & & & & & & & & & & & & & & \\
\hline Ad. ioides Zieg. & & 1 & & & & & & & & & & & & & & & \\
\hline Ad. nodosa Ul. \& Bas. & & 1 & & & & & & & & & & & & & & & \\
\hline Pa. brevis Zieg. \& Sand. & & 1 & & & & & & & & & & & & & & & \\
\hline Pa. gigas extensa Zieg. \& Sand. & & 3 & 1 & & & & & & & & & & & & & & \\
\hline Pa. hassi Müll. \& Müll. & & 1 & & & & & & & & & & & & & & & \\
\hline Pa. jamieae Zieg. \& Sand. & & 4 & & & & & & & & & & & & & & & \\
\hline Pa. linguiformis Müll. & & 4 & & & & & & & & & & & & & & & \\
\hline Pa. ljaschenkoae Ovn. & & 1 & & & & & & & & & & & & & & & \\
\hline Pa. juntianensis Han & & 1 & & & & & & & & & & & & & & & \\
\hline Pa. rhenana Bisch. & & 1 & & & & & & & & & & & & & & & \\
\hline Pa. subrecta Mill. \& Young. & & 1 & & & & & & & & & & & & & & & \\
\hline Pol. macilentus Ovn. \& Kuzm. & & 1 & 1 & & & & & & & & & & & & & & \\
\hline Ad. curvata (Br. \& M.) & & & 1 & & & & & & & & & & & & & & \\
\hline Anc. asymmetricus (UI. \& Bas.) & & & 1 & & & & & & & & & & & & & & \\
\hline Ic. alternatus alternatus $\mathrm{Br}$ \& $\mathrm{M}$. & 4 & 2 & 4 & 7 & 2 & 2 & 4 & 24 & 6 & & & 1 & 1 & 14 & 1 & & \\
\hline Pa. triangularis Sann. & & & & 1 & & & 2 & 5 & 1 & & & 1 & 2 & 3 & & & \\
\hline Ic. i. iowaensis Youn. \& Pet. & & & & & & & 1 & 2 & & & & & & & & & \\
\hline Ic. alternatus helmsi Sand. & & & & & & & & 4 & & & & & & & & & \\
\hline Pa. clarki Zieg. & & & & & & & & 1 & & & & 1 & & & & & \\
\hline Pa. q. praeterita Schül. & & & & & & & & 1 & & & & 1 & & & & & \\
\hline Pol. subinornatus Strel. & & & & & & & & 8 & & & & & 2 & & & & \\
\hline Pol. brevilaminus $\mathrm{Br}$ \& $\mathrm{M}$. & & & & & & & & & 1 & & & & & & & & \\
\hline Pa. glabra acuta Helms & & & & & & & & & & & & & & & & 12 & \\
\hline Pa. glabra glabra UI. \& Bas. & & & & & & & & & & & & & & & & 10 & \\
\hline Pa. glabra lepta Zieg. \& Hud. & & & & & & & & & & & & & & & & 10 & \\
\hline Pa. glabra prima Zieg. \& Hud. & & & & & & & & & & & & & & & & 22 & 2 \\
\hline Pa. m. marginifera Helms & & & & & & & & & & & & & & & & 8 & \\
\hline Pa. minuta minuta $\mathrm{Br} . \& \mathrm{M}$. & & & & & & & & & & & & & & & & 15 & 6 \\
\hline Pa.p. schindewolfi Müll. & & & & & & & & & & & & & & & & 7 & 2 \\
\hline Pa. stoppeli Sand. \& Zieg. & & & & & & & & & & & & & & & & 4 & \\
\hline Pol. glaber glaber UI. \& Bas. & & & & & & & & & & & & & & & & 9 & \\
\hline
\end{tabular}

considerably less than those of the genus Palmatolepis (see Table 1). The Barma shell stones in the conodont assemblage contain together with Palmatolepis triangularis Sann. 6 Frasnian conodont species of the genus Palmatolepis, which are found in Askyn Horizon (see Table 1). The signs of reworked shells brachiopods are absent in the Bol'shaya Barma section (Mizens 2007). Some Frasnian conodont and brachiopod taxa may have survived the F/F extinction (Schülke 1995, Racki et al. 2002, Abramova \& Artyushkova 2004).

The Barma shell stones are gradationally overlaid by light-grey thick-layered crinoid-brachiopod limestones of the Makarovo Horizon, with which they have a continuous sequence. The base of the Makarovo Horizon is characterized by a conodont assemblage of the Upper triangularis Zone (see Table 1).

The Akkyr section. - One of the best exposures of the F/F brachiopod shell limestones is located on the right bank of the Zilim River at the mouth of the Bol'shaya Kunderlya Rivulet (see Fig. 1A, B). A.N. Abramova (1999) called it the Akkyr section. The shell stones are $2.10 \mathrm{~m}$ thick. The lower $0.6 \mathrm{~m}$ interval has been assigned to the Askyn Horizon of the Frasnian. The upper $1.5 \mathrm{~m}$ interval has been identified as the Barma Horizon of the Famennian. 
Rezeda C. Tagarieva • Conodont biodiversity of the Frasnian-Famennian boundary interval, Southern Urals

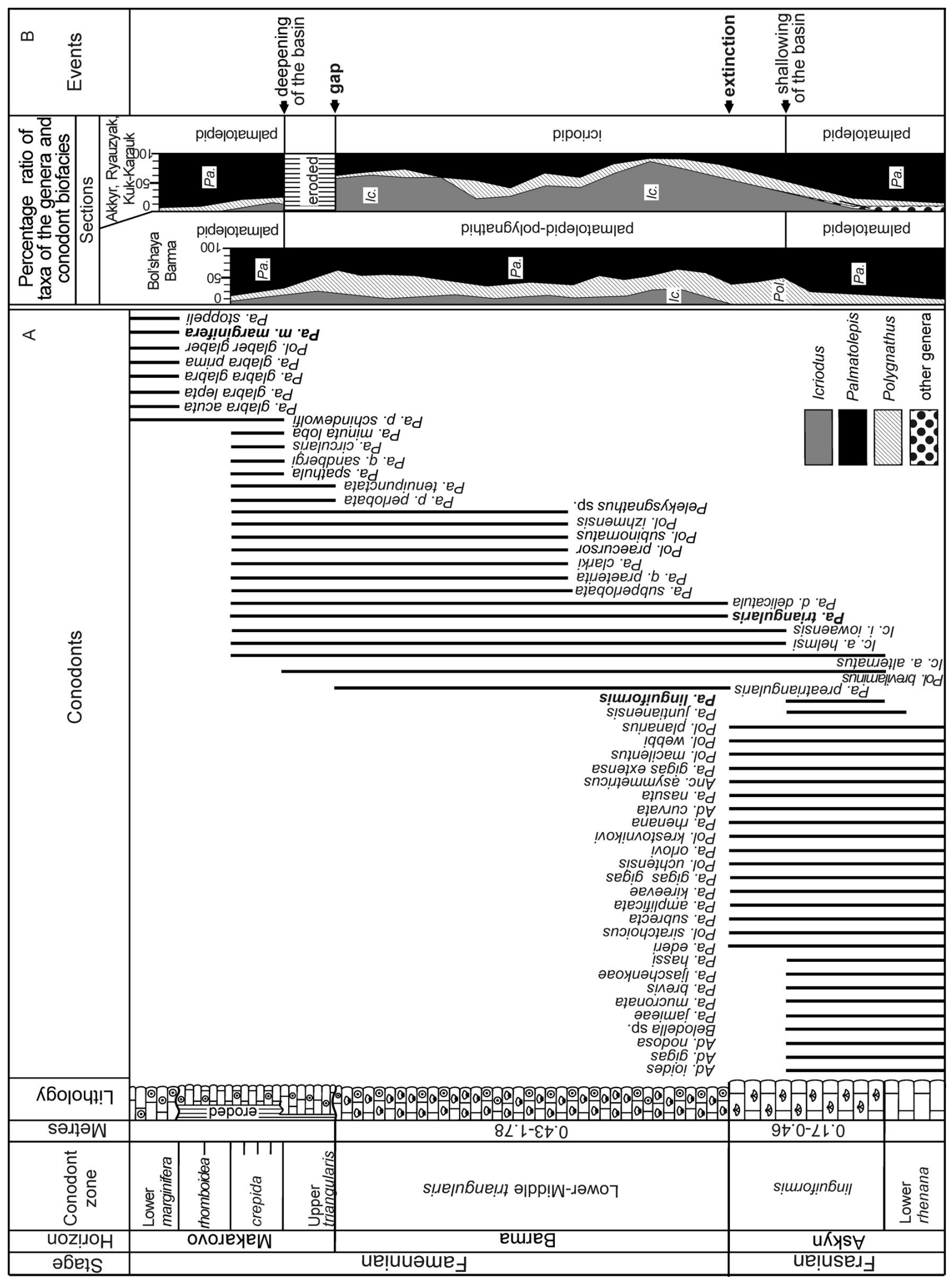


The structure of the Frasnian portion of these brachiopod shell limestones looks like an alternation between thin interbeds (1-2 cm) of light-grey crinoid and brachiopod varieties (see Fig. 3). The lower $0.25 \mathrm{~m}$ interval is characterized by conodonts of the Upper rhenana Zone. Taxonomically, the conodont assemblage is represented by 4 genera and 14 species (see Table 2). Representatives of the genus Palmatolepis dominate the other taxa. Among prevailing species are Palmatolepis nasuta Müll. and $P a$. subrecta Mill. \& Young.

In this section the thickness of the linguiformis Zone reaches $0.35 \mathrm{~m}$. The conodont assemblage is represented by 5 genera and 27 species (see Table 2). Species of the genus Icriodus make their appearance just in this interval, the number getting noticeably greater by the end of the linguiformis Zone. Near the F/F boundary the conodont assemblage becomes taxonomically much poorer.

In the Famennian portion the shell limestone (Barma Horizon) are characterized by denser clusters of brachiopods. There is no expressed stratification in the rock (see Fig. 3). Coquina is loose at the base. The matrix that binds brachiopod shells together consists of crinoid segments for the most part. Weak iron staining is visible.

At the base of the Barma Horizon the conodont assemblage contains rare individual specimens of Palmatolepis triangularis Sann. and Pa. praetriangularis Zieg. \& Sand. Along with them, one can find a great number of pass through the Famennian taxa of the genus Icriodus and to a smaller extent Polygnathus brevilaminus Br. \& M. (see Table 2). The diversity of brachiopods from the Barma coquinas in the Akkyr section is somewhat less than that in the Bol'shaya Barma section. However, the Akkyr section contains pentamerids which are absent in the stratotype (Mizens 2007).

The upper $1.12 \mathrm{~m}$ interval of the Barma Horizon is characterized by the occurrence of Palmatolepis clarki Zieg., Pa. delicatula delicatula Br. \& M., Pa. quadratinodosalobata praeterita Schül., Pa. subperlobata Br. \& M., Polygnathus praecursor Mat., Pol. subinornatus Strel. and Pelekysgnathus sp. (see Table 2).

Above the Barma coquinas the conodont succession displays a slight gap corresponding to the Upper triangularis Zone. Conodonts characteristic of the Lower crepida Zone are accumulated in the overlying thin-lay- ered crinoid-brachiopod limestones of the Makarovo Horizon (see Table 2).

The Ryauzyak section. - The Ryauzyak section is one of the few complete Upper Devonian sections. It is situated on the right bank of the Ryauzyak River, $5 \mathrm{~km}$ upstream from the village of Sargaevo (see Fig. 1). In this section the brachiopod shell limestone of the F/F interval is of the smallest thickness, as compared to the other sections, and is $0.60 \mathrm{~m}$ thick. The coquina is dark-grey; brachiopod shells are in close contact with each other, but for the most part they are recrystallized. The matrix is composed of small crinoid segments.

In this section the Frasnian portion of the brachiopod shell stone does not visually differ from the Famennian one, and the F/F boundary is determined only by the alternation of the conodont and brachiopod faunas.

The Frasnian includes the lower $0.15 \mathrm{~m}$ interval of the coquina corresponding to the linguiformis Zone. The zonal conodont association is characterized by a rich diversity represented 5 genera and 22 species (Table 3 ). The most abundant taxa is Palmatolepis nasuta Müll., the number of Belodella sp., Icriodus alternatus alternatus $\mathrm{Br} . \& \mathrm{M}$., Palmatolepis amplificata Klapp., Kuzm. \& Ovn., Pa. gigas extensa Zieg. \& Sand., Pa. rhenana Bisch., Pol. krestovnikovi Ovn., Pol. macilentus Ovn. \& Kuzm., Pol. siratchoicus Ovn. \& Kuzm. is 3 to 5 times less. The rest of the taxa are characterized by scarce specimens. The peculiar feature of the conodont assemblage in this section is the abundant presence of juvenile forms in the samples.

In this section the Barma Horizon has a small thickness of $0.45 \mathrm{~m}$. Similar to the rest of the sections, its base is determined by simultaneous occurrence of the conodont Palmatolepis triangularis Sann. and brachiopod Parapugnax markovskii (Yud.). The conodont assemblage from the lower part of the Barma Horizon $0.35 \mathrm{~m}$ thick is extremely poor and involves solitary specimens of Palmatolepis triangularis Sann., Pa. praetriangularis Zieg. \& Sand., Polygnathus brevilaminus Br. \& M., Icriodus alternatus alternatus $\mathrm{Br}$. \& M., Ic. alternatus helmsi Sand., Ic. iowaensis iowaensis Young. \& Pet. At the top of the Barma shell limestone the conodont biodiversity is complemented by the species Palmatolepis clarki Zieg., Pa. quadratinodosalobata praeterita Schül., Pa. subperlobata Br. \& M., Pol. subinornatus Strel. (see Table 3).

Figure 6. Conodonts from the Bol'shaya Barma, Akkyr and Kuk-Karauk sections, Upper Devonian, Frasnian, Askyn Horizon (all figures $\times$ 40). - A, B, E, F - Palmatolepis rhenana Bischoff; A, B, F - Akkyr section; A - Upper rhenana Zone, sample A-12a/4; B, F - linguiformis Zone, B - sample A-12a/5; E - sample A-12a/6; E - Kuk-Karauk section, linguiformis Zone, sample K-II-C/1. • C, D, G, H - Palmatolepis nasuta Müller; C, D, H - Akkyr section; C, D - Upper rhenana Zone; C - sample A-12a/1; D - sample A-12a/4; H - linguiformis Zone, sample A-12a/5; G - Kuk-Karauk section, linguiformis Zone, sample K-II-C/1. - I - Palmatolepis hassi Müller \& Müller, Bol'shaya Barma section, linguiformis Zone, sample B-1/8. - J, K, O - Palmatolepis subrecta Miller \& Youngquist, linguiformis Zone; J, K - Akkyr section, sample A-12a/5; O - Kuk-Karauk section, sample $\mathrm{K}-\mathrm{II}-\mathrm{C} / 1 . \bullet \mathrm{L}, \mathrm{M}-$ Palmatolepis amplificata Klapper, Kuz'min \& Ovnatanova, Akkyr section, linguiformis Zone, sample A-12a/5. • N - Palmatolepis ljaschenkoae Ovnatanova, Akkyr section, linguiformis Zone, sample A-12a/5. 
Rezeda C. Tagarieva • Conodont biodiversity of the Frasnian-Famennian boundary interval, Southern Urals

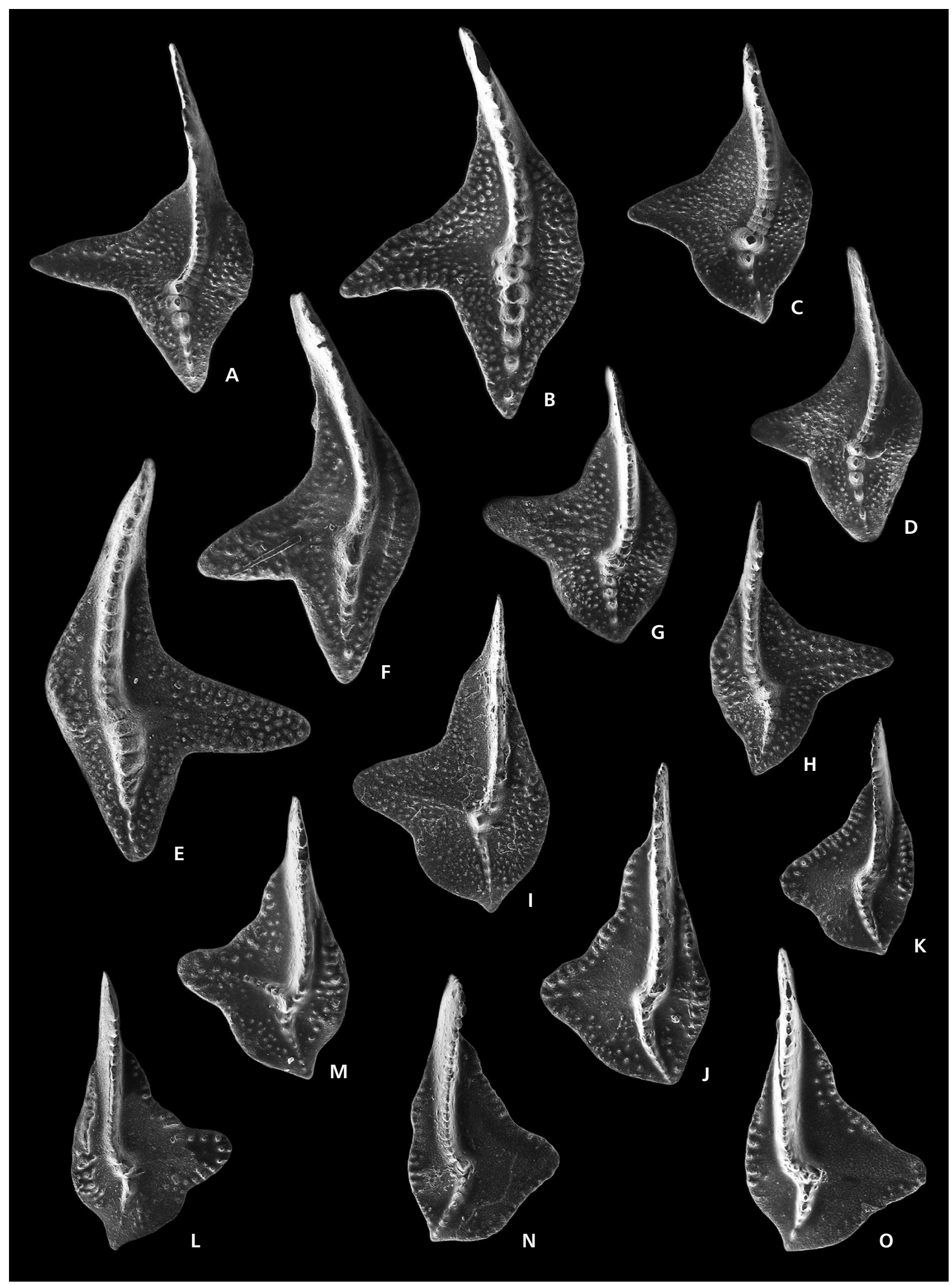


A short-time stratigraphic gap is observed above the shell stones of the Barma Horizon, and the overlying Makarovo Horizon begins with the Lower crepida Zone. The duration of the gap, as well as in the Akkyr section, corresponds to the interval of the conodont Upper triangularis Zone. The Makarovo deposits are represented by layered brownish-grey crinoid limestones, sometimes with iron staining. For the most part the conodont assemblage is characterized by the taxa of the genus Palmatolepis represented by abundant specimens perfectly preserved (see Table 3). The number of the representatives of the genus Icriodus becomes sharply reduced.

The Kuk-Karauk section. - The Kuk-Karauk section is the most southern one in the West Uralian Folded Zone where the boundary $\mathrm{F} / \mathrm{F}$ interval is represented by brachiopod shell limestone. It is located on the Sikaza River opposite the mouth of the Kuk-Karauk Rivulet (see Fig. 1A, B). This brachiopod shell stone is $0.7 \mathrm{~m}$ thick and characterized by a heterogeneous structure. Polished slab demonstrate repeated 2-3 cm interbedding of light-grey coquina with rather dense shell "packing" and loose crinoid limestone (Fig. 4). The lower part of brachiopod shell stones $0.20 \mathrm{~m}$ thick belongs to the linguiformis Zone. The assemblage includes 5 conodont genera and 20 species (Table 4). The conodont Palmatolepis nasuta Müll. is the most abundant taxon, corresponding to half of the whole conodont fauna. The smaller part is represented by Icriodus alternatus alternatus Br. \& M., Polygnathus siratchoicus Ovn. \& Kuzm. and others (see Table 4).

The thickness of the Famennian portion of the shell limestone is $0.50 \mathrm{~m}$; it encompasses the interval of the conodont Lower-Middle triangularis zones. The first solitary specimens of Palmatolepis triangularis Sann. are found just at the bottom of the Barma shell stones together with brachiopod Parapugnax markovskii (Yud.). The conodont assemblage of the Barma Horizon is extremely poor. The lower part of the horizon $0.15 \mathrm{~m}$ thick contains only the zonal index species Palmatolepis triangularis Sann. and Icriodus alternatus alternatus Br. \& M. Similar to the rest of the sections, the conodont biodiversity tends to increase in the upper part of the Barma coquina (see Table 4).
The shell stone of the Barma Horizon are overlaid by light-grey and yellow, grained, layered crinoid-coral limestones of the Makarovo Horizon with a stratigraphic gap that equals the interval of three conodont Upper triangularis-Upper rhomboidea zones. In the crinoid limestones immediately above the shell stones there are conodonts characteristic of the Lower marginifera Zone (see Table 4).

\section{Conodont biodiversity and biofacies}

The conodont biodiversity found in the brachiopod shell stone of the boundary $\mathrm{F} / \mathrm{F}$ interval is characterized by a rich taxonomic composition. Taxa of the genera Palmatolepis, Polygnathus and Icriodus form the basis for conodont assemblages in all the samples. These genera are considered to be the basic indicators of paleobasinal depths (Seddon \& Sweet 1971, Sandberg 1976, Sandberg \& Dreesen 1984, Dreesen et al. 1986, Sandberg et al. 1988, Ziegler \& Sandberg 1990). Other Upper Devonian genera occur in a markedly smaller number. In the sections under investigation an analysis has been performed for the conodont taxonomic and quantitative diversity in the boundary Frasnian/Famennian interval. The number of unbroken conodonts of the taxa encountered in each of the samples has been calculated.

In the Akkyr, Ryauzyak and Kuk-Karauk sections at the base of the brachiopod shell limestone of the Askyn Horizon of the Frasnian Stage the conodont assemblage is very rich in its diversity and includes 6 genera and 30 species (Fig. 5). The clear dominance belongs to palmatolepids that are represented by 16 taxa and correspond to the comprise 78 percent of the whole fauna (see Fig. 5B, Tables 2-4). Among characteristic species are Palmatolepis amplificata Klapp., Kuzm. \& Ovn., Pa. gigas extensa Zieg. \& Sand., Pa. hassi Müll. \& Müll., Pa. jamieae Zieg. \& Sand., Pa. juntianensis Han, Pa. nasuta Müll., Pa. rhenana Bisch. and Pa. subrecta Mill. \& Young. (Figs 6, 7). The abundance of Palmatolepis nasuta Müll. is noted almost in each sample whereas the taxa of the genus Polygnathus are half as many. Representatives of the genera Ancyrodella, Ancyrognathus, Belodella and Icriodus are very rare in the assemblage amounting only to 11 percent (see Tables 2-4).

Figure 7. Upper Devonian, Frasnian, Askyn Horizon conodonts (all figures $\times 50$ ). • A, B - Palmatolepis linguiformis Müller, linguiformis Zone; A - Ryauzyak section, sample R-b; B - Akkyr section, sample A-12a/5. • C, D - Palmatolepis gigas extensa Ziegler \& Sandberg, linguiformis Zone; C - Kuk-Karauk section, sample K-II-C/1; D - Ryauzyak section, sample R-b. • E - Palmatolepis beckeri Klapper, Akkyr section, Upper rhenana Zone, sample A-12a/4. • F-J - Palmatolepis juntianensis Han, F, G, I, J - Akkyr section; J - Upper rhenana Zone, sample A-12a/4; G, I, J - linguiformis Zone, sample A-12a/5; H - Kuk-Karauk section, linguiformis Zone, sample K-II-C/1. • K, L - Palmatolepis aff. nicolli Klapper, Akkyr section, linguiformis Zone, sample A-12a/5. $・ \mathrm{M}, \mathrm{N}$ - Ancyrodella curvata (Branson \& Mehl), linguiformis Zone; M - Ryauzyak section, sample R-b; N - Akkyr section, sample A-12a/6. • O - Ancyrodella gigas Youngquist, linguiformis Zone, R-b. • P - Palmatolepis sp., Akkyr section, linguiformis Zone, sample A-12a/5. - Q - Icriodus alternatus alternatus Branson \& Mehl, Akkyr section, linguiformis Zone, sample A-12a/5. $\bullet$ R - Icriodus iowaensis iowaensis Youngquist \& Peterson, Akkyr section, Upper rhenana Zone, sample A-a/3. • S - Ancyrodella ioides Ziegler, Akkyr section, Upper rhenana Zone, sample A-12a/1. - T - Polygnathus macilentus Ovnatanova \& Kuzmin, Akkyr section, linguiformis Zone, sample A-12a/5. • U, V - Polygnathus brevilaminus Branson \& Mehl, Akkyr section, linguiformis Zone; U - sample A-12a/5; V - sample A-a/3. 
Rezeda C. Tagarieva • Conodont biodiversity of the Frasnian-Famennian boundary interval, Southern Urals

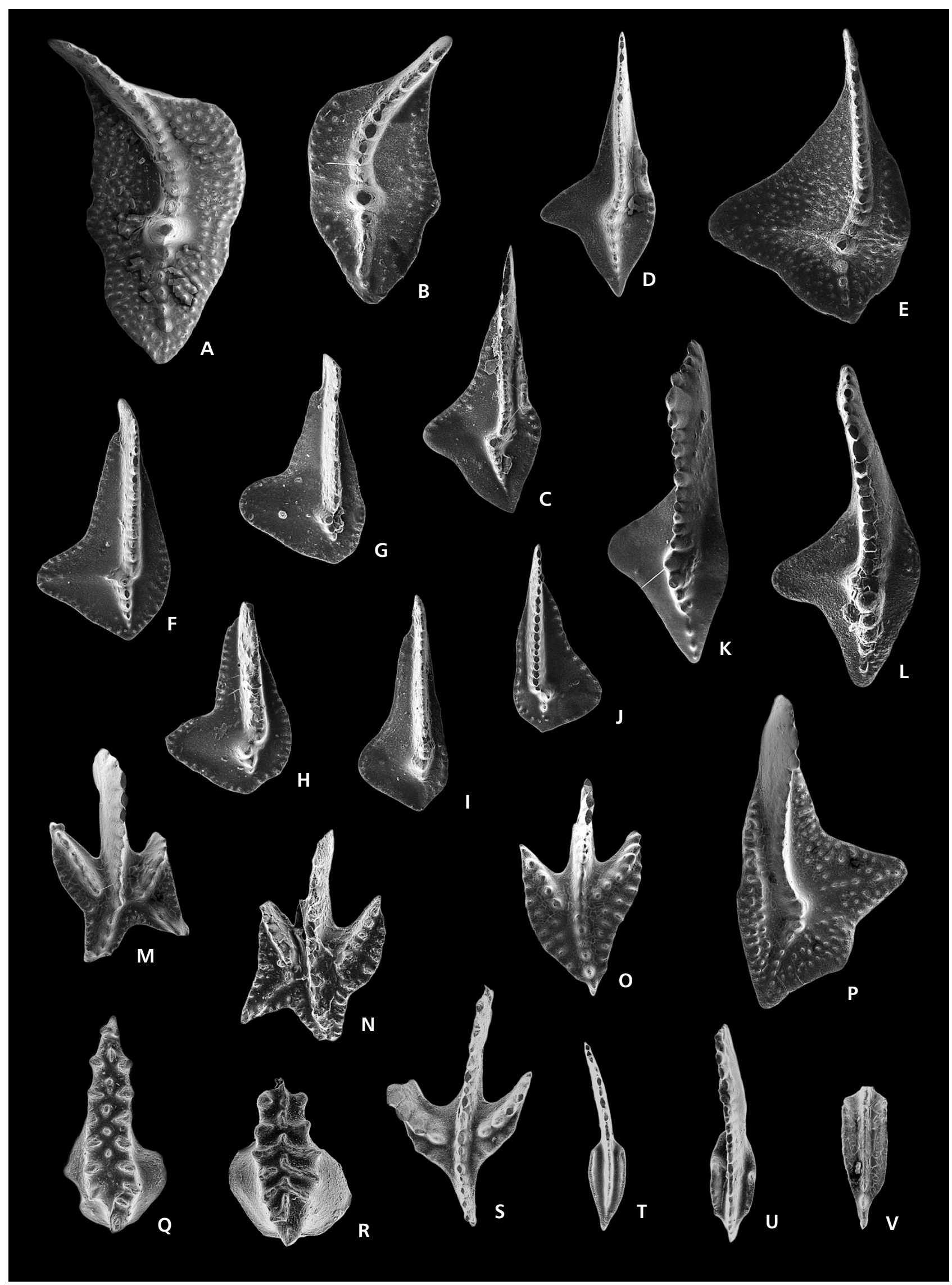


This conodont assemblage characterizes the palmatolepid (pelagic) biofacies (see Fig. 5B; Sandberg 1976, Ziegler \& Dreesen 1984, Sandberg et al. 1988, Zielger \& Sandberg 1990).

In the upper part of the Askyn coquina (the linguiformis Zone), these sections show a considerable reduction in the conodont diversity (see Tables 2-4). The number of taxa of the genus Palmatolepis gets halved, and a sudden reduction of their quantity to 23 percent is observed (see Fig. 5A, B). The quantity of the Icriodus taxa increases abruptly $(52 \mathrm{pc})$, though they are taxonomically represented by three species only: Icriodus alternatus alternatus Br. \& M., Ic. alternatus helmsi Sand. and Ic. iowaensis iowaensis Young. \& Pet. Thus, at the end of the Frasnian time, near the F/F boundary the palmatolepid (pelagic) biofacies gives way to the icriodid (shallow water) one (see Figs 5A, B, 6, 7, Tables 2-4).

Among the sections under investigation the Bol'shaya Barma section has its own peculiarities. Here, the Icriodus taxa are absent in the Frasnian brachiopod shell stone (the linguiformis Zone), and the assemblage contains representatives of the genera Palmatolepis and Polygnathus. They are almost in the equal ratio (53 and $45 \mathrm{pc}$ ) and form the palmatolepid-polygnathid (relatively deep water) biofacies (see Fig. 5B, Table 1).

Along with the conodonts all the sections under investigation contain fish remains, such as Bradiodontidae gen. indet., Acanthodei gen. indet., Crossopterigii gen. indet., Elasmobranchii gen. indet., Haplacanhus sp., Phoebodus sp., Cladodus sp., Onychodus sp., Cobelodus sp. (Abramova 1999, Artyushkova et al. 2011a).

In the uppermost Askyn Horizon, close to the F/F boundary, Frasnian species of the genera Palmatolepis die out, and the genera Ancyrodella and Belodella become totally extinct. Icriodus alternatus alternatus $\mathrm{Br}$. \& M., Ic. alternatus helmsi Sand., Ic. iowaensis iowaensis Young. \& Pet. and Polygnathus brevilaminus Br. \& M. pass through the Famennian (see Fig. 5A, Tables 2-4). An identical situation is observed in the brachiopod assemblages. Thus, the genera
Devonoproductus, Hypothyridina, Calvinaria, Septalaria, Koltubania, Atryparia, Theodossia, Squmulariina, Tecnocyrtina and representatives of the orders Pentamerida and Atrypida become extinct (Rzhonsnitskaya et al. 1998; Abramova 1999; Mizens 2007, 2009).

Abrupt changes that occurred in the taxonomic structure of the conodont assemblages at the end of the Frasnian time affected the conodont biodiversity of the early Famennian age. At the base of the Barma Horizon the conodont association is extremely poor and uniform. The genus Palmatolepis is represented by solitary specimens of Palmatolepis triangularis Sann., Pa. delicatula delicatula Br. \& M. and Pa. praetriangularis Zieg. \& Sand. (see Figs 5A, B, 8, Tables 2-4).

In the Akkyr, Ryauzyak and Kuk-Karauk sections the dominance of species of the genus Icriodus can be observed Icriodus alternatus alternatus $\mathrm{Br}$. \& $\mathrm{M}$. and to a smaller extent Ic. alternatus helmsi Sand., Ic. iowaensis iowaensis Young. \& Pet., the number of which reaches a total of 80 percent. The conodont association of the lower part of the Famennian can be assigned to the icriodid (shallow water) biofacies (see Fig. 5A, B, Tables 2-4).

In the Bol'shaya Barma section the taxa of the genera Palmatolepis and Polygnathus continue to prevail beginning with the base of the Barma Horizon, however, the assemblage is complemented with rare specimens of Icriodus that make their first appearance in this section (see Table 1). Beginning with the Famennian age, the conodont association in this section is characterized palmatolepidpolygnathid (relatively deep water) biofacies (see Fig. 5B).

The enrichment of the assemblages with new species, such as Palmatolepis clarki Zieg., Pa. quadratinodosalobata praeterita Schül., Pa. subperlobata Br. \& M., Polygnathus izhmensis Kuzm., Pol. praecursor Mat., Pol. subinornatus Strel. (see Fig. 8) is observed far above the Frasnian/Famennian boundary, the span corresponds to $0.42 \mathrm{~m}$ in the Bol'shaya Barma section, $0.38 \mathrm{~m}$ in the Akkyr section, $0.33 \mathrm{~m}$ in the Ryauzyak section and $0.15 \mathrm{~m}$ in the Kuk-Karauk section (see Tables 1-4).

Figure 8. Upper Devonian, Famennian conodonts $(\mathrm{A}-\mathrm{M} \times 60, \mathrm{~N}-\mathrm{W} \times 50)$. $-\mathrm{A}-\mathrm{E}, \mathrm{H}-$ Palmatolepis triangularis Sannemann, Barma Horizon, Lower-Middle triangularis zones; A, B - Ryauzyak section, sample R-3; C, D, H - Akkyr section; C, H - sample A-b/2; D - sample A-18d. - E - Bol'shaya Barma section, sample 02357-1. • F, G, K - Palmatolepis quadratinodosalobata praeterita Schülke, Barma Horizon, Lower-Middle triangularis zones; F-K - Akkyr section; sample A-18d; G - Bol'shaya Barma section, sample 02358. • I, L, M - Palmatolepis clarki Ziegler; I, M - Akkyr section, Barma Horizon, Lower-Middle triangularis zones, sample A-18a; L - Ryauzyak section, Makarovo Horizon, Lower crepida Zone, sample R-6. • J - Palmatolepis delicatula delicatula Branson \& Mehl, Akkyr section, Makarovo Horizon, Lower crepida Zone, A-19b. • N-O - Icriodus iowaensis iowaensis Youngquist \& Peterson, Barma Horizon, Lower-Middle triangularis zones; N - Akkyr section, sample A-14a; O - Ryauzyak section, sample R-e. $\bullet$ P - Icriodus alternatus alternatus Branson \& Mehl, Akkyr section, Barma Horizon, Lower-Middle triangularis zones, sample A-14a. - Q - Palmatolepis circularis Szulczewski, Ryauzyak section, Makarovo Horizon, Lower crepida Zone, sample R-6. • R - Palmatolepis glabra lepta Ziegler \& Huddle, Kuk-Karauk section, Makarovo Horizon, Lower marginifera Zone, sample K-II-F. • S, T - Polygnathus brevilaminus Branson \& Mehl, Barma Horizon, Lower-Middle triangularis zones, S - Ryauzyak section, sample R-3; T - Bol'shaya Barma section, sample 02357-1. - U - Polygnathus izhmensis Kuzmin, Ryauzyak section, Barma Horizon, Lower-Middle triangularis zones, sample R-e. • V - Palmatolepis wolskae Ovnatanova, Akkyr section, Makarovo Horizon, Lower crepida Zone, A-19b. • W - Palmatolepis stoppeli Sandberg \& Ziegler, Kuk-Karauk section, Makarovo Horizon, Lower marginifera Zone, sample K-II-F. 


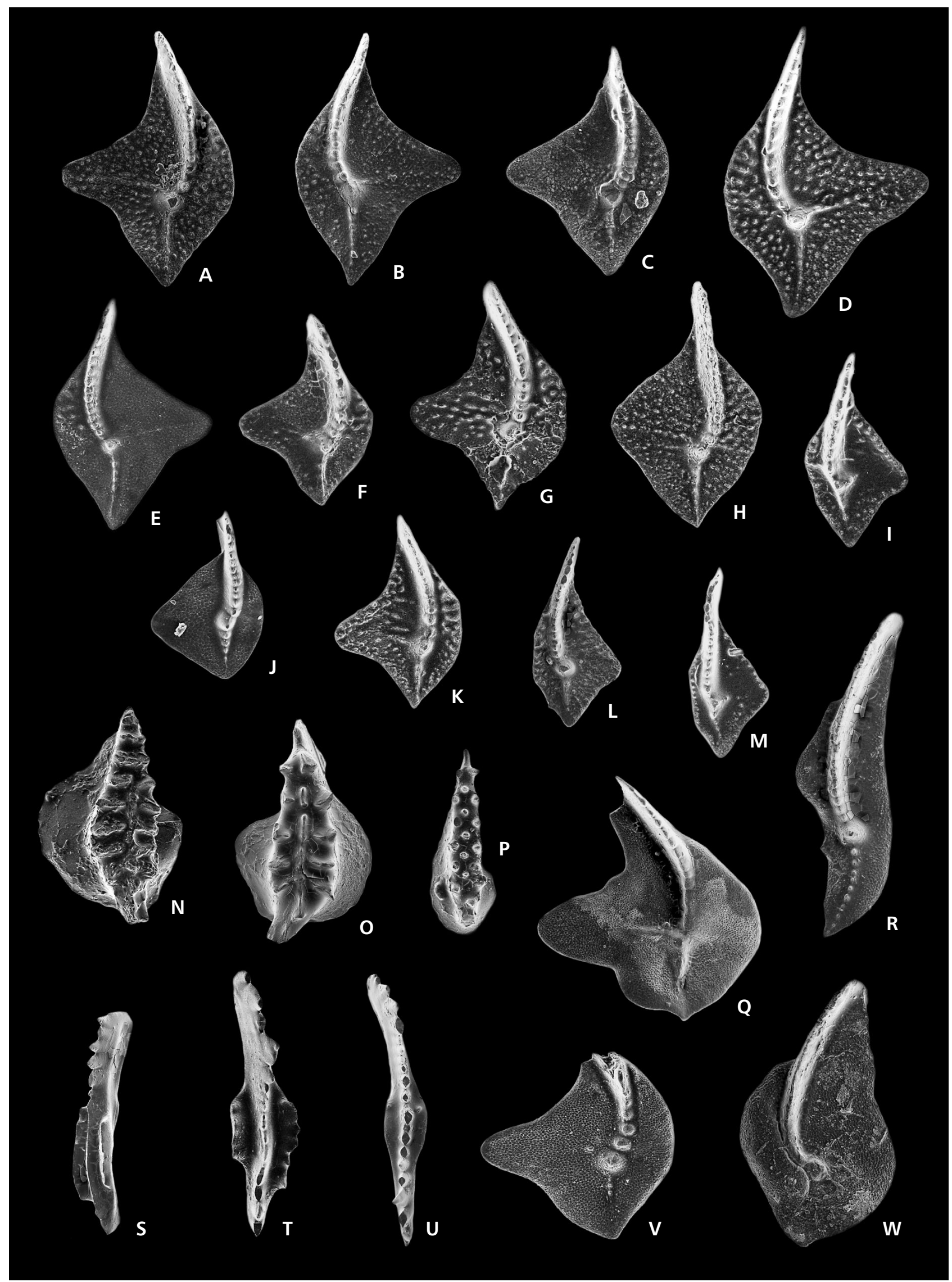


Above the Barma coquina a complete conodont succession has been established only in the Bol'shaya Barma section. The beginning of the Makarovo Horizon base is noted for the appearance of species Palmatolepis perlobata perlobata U1. \& Bas., Pa. spathula Schül. and Pa. tenuipunctata Sann. characteristic of the Upper triangularis Zone (Barskov et al. 1987, Ziegler \& Sandberg 1990, Schülke 1995; see Table 1). The ratio among the taxa of the genera Palmatolepis, Polygnathus and Icriodus does not change, and the palmatolepid-polygnathid (relatively deep water) biofacies is still preserved in this interval (see Fig. 5B). The appearance of the species $\mathrm{Pa}$. quadratinodosalobata sandbergi Ji \& Ziegl. and Pa. wolskae Ovn. in the Bol'shaya Barma section characterizes the Lower crepida Zone (Barskov et al. 1987, Ziegler \& Sandberg 1990, Schülke 1995; see Table 1). In the conodont assemblage an abrupt increase in the number of elements belonging to the genus Palmatolepis, up to the 75 percent of the whole conodont fauna, can be noticed. The palmatolepid-polygnathid (relatively deep water) biofacies of the Upper triangularis Zone gives way to the palmatolepid (pelagic) biofacies in the Lower crepida Zone (see Fig. 5B).

In the Akkyr and Ryauzyak sections the stratigraphic sequence does not yield any conodont indicative of the Upper triangularis Zone; here, the Makarovo Horizon begins with the Lower crepida Zone (see Tables 2, 3). The conodont association of the Lower crepida Zone, as in the Bol'shaya Barma section, is characterized by an abrupt increase in the number of the Palmatolepis taxa (69 pc) and corresponds to the palmatolepid (pelagic) biofacies (see Figs 5A, B, 8).

Only the upper part of the Makarovo Horizon in the scope of the conodont Lower marginifera Zone was determined in the Kuk-Karauk section above the Barma coquina. The richest taxonomic composition of conodonts is represented by species of the genus Palmatolepis and thus can be attributed the palmatolepid (pelagic) biofacies (see Figs 5A, B, 8, Table 4).

\section{Conclusions}

In the sections of the West Uralian Folded Zone (Bol's shaya Barma, Akkyr, Ryauzyak and Kuk-Karauk) the F/F boundary interval is represented by brachiopod shell limestone. According to conodonts, there can be recognized the Frasnian (upper part of the Askyn Horizon) and Famennian (Barma Horizon) portions of the shell stones, in which the detailed zonal conodont-based subdivision and establishment of biofacies have been performed.

At the top of the brachiopod coquina of the Askyn Horizon of the Frasnian (the linguiformis Zone) all the sections under investigation display much poorer conodont biodiversity; at the F/F boundary the majority of Frasnian conodont and brachiopod taxa become totally extinct. At the end of the Frasnian age abundant specimens of the Palmatolepis taxa that represent the pelagic (palmatolepid) biofacies are gradually replaced by the Polygnathus and Icriodus taxa of the shallow-water (icriodid) biofacies. Judging from the changes in the composition of the brachiopod assemblage at the end of the Frasnian age, it can be assumed that the basin was characterized by an active hydrodynamic regime with slight muddiness of ground (Mizens 2009). It is quite possible that observable restructurings in the conodont and brachiopod communities result from regression of the basin at the F/F boundary (Johnson et al. 1985; Johnson \& Sandberg 1988; Sandberg et al. 1988; Veimarn et al. 1998, 2004; Racki 1998; House et al. 2000; Racki et al. 2002; Yudina et al. 2002).

Beginning with the Famennian time after extinction of the majority of the Frasnian taxa, the recovery of the conodont faunal community proceeded at a very slow pace. The conodont assemblage of the Barma Horizon (Lower-Middle triangularis zones) is taxonomically poor and it can be assigned to shallow-water conodont biofacies. An abrupt increase in the conodont biodiversity is seen only in the Lower crepida and especially during to Lower marginifera zones corresponding to the deposition of the Makarovo Horizon.

At the $\mathrm{F} / \mathrm{F}$ boundary in this region there occurs marked tendency to shallowing of the basin with its maximum in the Lower-Upper triangularis zones. This is evidenced by stratigraphic gaps determined by means of conodonts above the linguiformis Zone (Veimarn et al. 1998, 2004; Abramova 1999; Artyushkova et al. 2011a). In most sections known on the western slope of the South Urals (Gabdyuk, Mendym, Zigan and others) the range of this gap varies from one zone to several conodont zones (Abramova 1999). In those sections where the F/F boundary is determined within brachiopod shell stones the gap is observed above the Barma Horizon (except the Bol'shaya Barma section) and encompasses the interval corresponding to the conodont Upper triangularis Zone in the Akkyr and Ryauzyak sections and to the Upper triangularis-Upper rhomboidea zones in the Kuk-Karauk section.

The Frasnian/Famennian boundary is marked with dark hypoxic limestone and the Upper Kellwasser Mass Extinction Event (Walliser et al. 1986, Klapper et al. 1993, Walliser 1996, Alekseev 1998, Veimarn et al. 1998). In the sections on the western slope of the South Urals the F/F boundary interval is different and characterized by thin brachiopod shell limestones (Bol'shaya Barma, Akkyr, Ryauzyak, Kuk-Karauk and Kuktash sections) and stratigraphic gaps (Gabdyuk, Mendym, Zigan and others sections). The Frasnian/Famennian boundary revealed mass extinction of the main Frasnian conodont and brachiopod taxa (Rzhonsnitskaya et al. 1998; Abramova 1999; 
Abramova \& Artyushkova 2004; Mizens 2007, 2009; Tagarieva 2010). The bio-event is represents a significant regional event that is correlated with the Upper Kellwasser Mass Extinction Event.

\section{Acknowledgements}

The author would like to express the deep gratitude to her research supervisor Olga V. Artyushkova for the most valuable recommendations and critical comments during the preparation of the paper. Also, the author would like to thank Lyudmila I. Kononova (Faculty of Geology at Moscow State University, Moscow) and Nadezhda G. Izokh (Institute of Petroleum Geology and Geophysics, Siberian Division, RAS, Novosibirsk) for consultations on conodonts. Brachiopods have been studied by Anita G. Mizens and Larisa I. Mizens (Institute of Geology and Geochemistry, Ural Division, RAS, Ekaterinburg), to whom the author wishes to give her sincere thanks. Great help was provided in photographing the conodonts by Nadezhda G. Izokh and Elena M. Kirilishina (Earth Science Museum, Moscow State University, Moscow). The author is grateful to Norman Savage and Claudia Spalletta for review of the manuscript and useful comments and suggestions. The investigation was financially supported by the Russian Foundation for Basic Research (RFBR), grants 11-05-01105-a and 11-05-00737-a. This study is a contribution to the IGCP 596 Project.

\section{References}

ABramova, A.N. 1992. The Frasnian/Famennian boundary in the South Urals, 1-2. International Symposium on Devonian System and Its Economic Oil and Mineral Resources, Abstracts. Guilin, China.

Abramova, A.N. 1999. Frasnian stage at the western slope of the South Urals. 54 pp. Institute of Geology, Ufa Scientific Center, Russian Academy of Sciences, Ufa. [in Russian]

Abramova, A.N. \& Artyushrova, O.V. 2004. The FrasnianFamennian boundary in the South Urals. Geological Quarterly 48(3), 217-232.

Alekseev, A.S. 1998. Mass extinctions in the Phanerozoic time. 76 pp. Synopsis for doctoral thesis, Moscow State University, Moscow. [in Russian]

Antsygin, N.Y., Popov, B.A. \& Chuvashov, B.I. (eds) 1993. Stratigraphic schemes of the Urals. $151 \mathrm{pp}$. Ural Branch Russian Academy of Sciences, Yekaterinburg. [in Russian]

Artyushrova, O.V., Maslov, V.A., Pazukhin, V.N., Kulagina, E.I., Tagarieva, R.C., Mizens, L.I. \& Mizens, A.G. 2011a. Devonian and Lower Carboniferous type sections of the western South Urals: Pre-Conference Field Excursion Guidebook. International Conference "Biostratigraphy, Paleogeography and Events in Devonian and Lower Carboniferous", Ufa, Sterlitamak, Russia, July 20-25, 2011. 92 pp. Ufa.

Artyushrova, O.V., Tagarieva, R.C. \& Mizens, A.G. 2011b. The Barma Beds as a biostratigraphic marker of the Famennian base in the South Urals. Biostratigraphy, paleogeography and events in Devonian and Lower Carboniferous
(SDS/IGCP 596 joint field meeting): Contributions of International Conference in memory of Evgeny A. Yolkin. Ufa, Novosibirsk, Russia, July 20 - August 10, 2011, 22-24. Publication House of SB Russian Academy of Sciences, "Geo Branch", Novosibirsk.

Barskov, I.S., Alekseev, A.S., Kononova, L.I. \& Migdisova, A.V. 1987. In Menner, V.V. (ed.) Atlas of Upper Devonian and Carboniferous conodonts. 144 pp. Moscow State University, Moscow. [in Russian]

Baryshev, V.N. \& Abramova, A.N. 1996. Conodont zonal subdivision of the Upper Devonian deposits on the western slope of the South Urals. Annual Report 1996, 26-30. Institute of Geology, Ufa Scientific Center, Russian Academy of Sciences, Ufa. [in Russian]

Breyvel, M.G., Papulov, G.N. \& Khodalevich, A.N. (eds) 1980. Stratigraphic schemes of the Urals. 152 pp. Sverdlovsk. [in Russian]

Domrachev, S.M. 1952. Devonian of the West Preuralian. Devonian of the Karatau Ridge and adjacent regions of the South Urals. Trudy Vsesoyuznogo neftyanogo nauchno-issledovatelskogo geologorazvedochnogo instituta (VNIGRI)/ Proceedings of All-Union Scientific Research Geology and Prospect Institute, New Series 61, 5-121. [in Russian]

Dreesen, R., SANDberg, C.A. \& Ziegler, W. 1986. Review of Late Devonian and Early Carboniferous conodont biostratigraphy and biofacies models as applied to the Ardenne Shelf, 27-42. In Bless, M.J.M. \& STREEL, M. (eds) Late Devonian events around the Old Red Continent. Annales de la Société Géologique de Belgique 109.

House, M.R., Menner, V.V., Becker, R.T., Klapper, G., OvnaTANOVA, N.S. \& KuZmin, V. 2000. Reef episodes, anoxia and sea-level changer in the Frasnian of the southern Timan (NE Russian Platform), 147-176. In Insalaco, E., Skelton, P.W. \& Palmer, T.J. (eds) Carbonate Platform Systems: Components and Interactions. Geological Society of London, Special Publications 17.

Izokh, O.G. \& MizEns, A.G. 2009. First evidence on carbon isotopic variations within the Frasnian/Famennian deposits of the South Urals. Proceedings of the $4^{\text {th }}$ Russian Conference on Isotopic Geochronology "Isotopic Systems and Time of Geological Processes" (June 2-4, 2009), 211-212. Institute of Precambrian Geology and Geochronology, Russian Academy of Sciences, Saint Petersburg. [in Russian]

Khodalevich, A.N. (ed.) 1968. Stratigraphic schemes of the Urals. 154 pp. Leningrad. [in Russian]

Klapper, G., Feist, R., Becker, R.T. \& House, M.R. 1993. Definition of the Frasnian/Famennian stage boundary. Episodes 16, 433-440.

Johnson, J.C., Klapper, G. \& SAndBerg, C.A. 1985. Devonian eustatic fluctuations in Euramerica. Geological Society of America Bulletin 96(5), 567-587.

DOI 10.1130/0016-7606(1985)96<567:DEFIE >2.0.CO;2

Johnson, J.C. \& SANDBERG, C.A. 1988. Devonian eustatic events in the Western United States and their biostratigraphic responses, 171-178. In McMillan, N.J., Embry, A.F. \& GLass, D.J. (eds) The Devonian of the World: Proceedings of the Second International Symposium on the Devonian System, Calgary, Canada. Volume III: Paleoecology and Biostratigraphy. 
Markovsky, B.P. 1948. Overview on the Devonian stratigraphy of the western slope of the Middle and South Urals. Proceedings of All-Union Scientific Research Geology and Prospect Institute, New Series 8, 22-28. Gosgeolizdat Publication, Leningrad. [in Russian]

Mizens, A.G. 2007. The brachiopod complexes from Frasnian/Famennian boundary sediments of the sections Bol'shaya Barma and Akkyr of the South Urals (Stratotype and Parastratotype of the Barma Beds). Lithosphere 6, 93-110. [in Russian]

Mizens, A.G. 2009. Upper Devonian brachiopods and biostratigraphy of the Middle and South Urals. $18 \mathrm{pp}$. Synopsis for Ph.D. thesis (Geology and Mineralogy), Novosibirsk. [in Russian]

NALIVKIN, D.V. 1926. On geological structure of the South Urals. Annales de l'Institut des Mines a Leningrade 7, 71-95. [in Russian]

Nalivkin, D.V. 1931. The Paleozoic of the western slope of the South and Middle Urals. Geological map, scale: 1:1,000,000. Explanatory note. [in Russian]

RACKI, G. 1998. Frasnian-Famennian biotic crisis: undervalued tectonic control? Palaeogeography, Palaeoclimatology, Palaeoecology 141, 177-198. DOI 10.1016/S0031-0182(98)00059-5

Racki, G., Racka, M., Matyja, H. \& Devleeschouwer, X. 2002. The Frasnian-Famennian boundary in the South Polish-Moravian shelf basins: integrated event-stratigraphical approach. Palaeogeography, Palaeoclimatology, Palaeoecology 181, 251-297. DOI 10.1016/S0031-0182(01)00481-3

Rzhonsnitskaya, M.A., Markovskit, B.P., Yudina, Y.A. \& SoKIRAN, E.V. 1998. Late Frasnian Atrypida (Brachiopoda) from the South Urals, South Timan and Kuznetsk Basin (Russia). Acta Palaeontologica Polonica 43(2), 305-344.

Schülke, I. 1995. Evolutive Prozesse bei Palmatolepis in der frühen Famenne-Stufe (Conodonta, Oder-Devon). Göttinger Arbeiten zur Geologie und Paläontologie 67, 1-108.

SANDBERG, C.A. 1976. Conodont biofacies of Late Devonian Polygnathus styriacus Zone in western United States, 171-186. In BARNES, C.R. (ed.) Conodont paleocology. Geological Association of Canada Special Paper 15.

SAndBerg, C.A. \& Dreesen, R. 1984. Late Devonian icriodontid biofacies, models and alternate shallow water zonation, 143-178. In CLARK, D.L. (eds) Conodont biofacies and provincialism. Geological Society of America Special Paper 196.
Sandberg, C.A., Ziegler, W., Dreesen, R. \& Butler, J.L. 1988. Late Frasnian mass extinction: conodont event stratigraphy, global changes, and possible causes, 263-307. In ZIEGLER, W. (ed.) $1^{\text {st }}$ International Senckenberg Conference and $5^{\text {th }}$ European Conodont Symposium (ECOS V) Contribution I. Courier Forschungsinstitut Senckenberg 102.

Seddon, G. \& SweEt, W.C. 1971. An ecological model for conodonts. Journal of Paleontology 45, 896-880.

Tagarieva, R.C. 2011. Conodonts from the Fransian-Famennian boundary deposits of Akkyr section (western slope of South Urals), 41-51. In Modern paleontology: Classical and new methods - 2011. Paleontological Institute of Russian Academy of Sciences, Moscow. [in Russian]

Veimarn, A.B., Naidin, D.P., Kopaevich, L.F., Alekseev, A.S. \& NazARov, M.A. 1998. Analysis of global catastrophic events under detailed stratigraphic investigations. $190 \mathrm{pp}$. Moscow State University Publishing House, Moscow. [in Russian]

Veimarn, A.B., Puchkov, V.N., Abramova, A.N., Naidin, D.P., Artyushrova, O.V., Baryshev, V.N., Degtyaryov, K.E., Kononova, L.I., Maslov, V.A., Mosejchuk, V.M., Pazukhin, V.N., Pravikova, N.V., Tevelev, A.V. \& Yarkova, A.V. 2004. Stratigraphy and geological event at the FrasnianFamennian boundary in the South Urals. Geological Quarterly 48(3), 233-244.

WALLISER, O.H. 1986. Towards a more critical approach to bioevents, 5-16. In WALLISER, O.H. (ed.) Global bio-events, Lecture Notes in Earth Sciences 8. Springer Verlag, Berlin \& Heidelberg.

WALLISER, O.H. 1996. Global events in the Devonian and Carboniferous, 225-250. In WALLISER, O.H. (ed.) Global Events and Event Stratigraphy in the Phanerozoic. Springer Verlag, Berlin.

Yudina, A.B., Racki, G., Savage, N.M., Racka, M. \& MalkowSKI, K. 2002. The Frasnian-Famennian events in a deep-shelf succession, Subpolar Urals: biotic, depositional and geochemical records. Acta Palaeontologica Polonica 47(2), 355-372.

ZIEGLER, W. \& SANDBERG, C.F. 1990. The Late Devonian Standart Conodont Zonation. Courier Forschungsinstitut Senckenberg $121,1-115$.

ZhамоуDA, A.I. 2008. Resolutions of Interdepartmental stratigraphic committee and its permanent commissions $38.131 \mathrm{pp}$. All-Russian Institute of Geological Research, Saint Petersburg. [in Russian] 